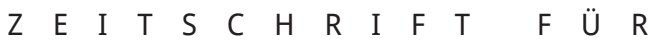

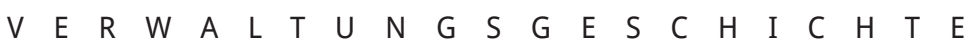

B A N D 2, 200717

$S$ E I T E $3-28$

D O I : $10.2478 /$ A DHI-2018-0013

\title{
Staat, Verwaltung und Raum im langen 19. Jahrhundert
}

Einleitung

\author{
STEFAN NELLEN, THOMAS STOCKINGER
}

\section{Ein Spannungsfeld mit drei Polen}

Dass zu den konstitutiven Merkmalen des >modernen Staates ein bestimmtes Verhältnis zum Raum gehört, ist ein Gemeinplatz nahezu sämtlicher Versuche, diesen Staat theoretisch und definitorisch zu fassen. Der Gedanke findet sich an prominenter Stelle in der klassischen deutschen Staatsrechtslehre des späten 19. und des beginnenden 20. Jahrhunderts mit ihrer Merkmalstrias von Staatsvolk, Staatsgebiet und Staatsgewalt; ${ }^{1}$ ebenso bei Max Weber, der in seiner berühmten, auf das Monopol der legitimen Gewaltanwendung abhebenden Definition explizit hervorhob, dass das "Gebiet « dabei als wesentliches Element zu verstehen sei. ${ }^{2}$ Auch noch in jenen viel späteren Modifikationen von Webers Formel, die diese relativieren, um sie von ihrer ausschließlichen Fixierung auf den europäisch-atlantischen Staat der >Moderne $\mathbf{z u}$ befreien, ${ }^{3}$ bleibt dieser Bezug auf den Raum des Staates unverzichtbar. Er begegnet aber auch in aktuellsten $\mathrm{Zu}$ gängen zur Staatlichkeit, die sich viel deutlicher von Weber abgrenzen, wenn etwa Patrick Joyce die Verbindung (»connecting «) von Orten, Dingen und Menschen durch Kommunikationstechnologien als eine Integration räumlicher Einheiten ${ }^{4}$ und zugleich als einen der fundamentalen Vorgänge der Staatsbildung beschreibt. ${ }^{5}$
Aus der ubiquitären Erwähnung des Zusammenhangs von Staat und Raum lassen sich freilich zwei entgegengesetzte Schlüsse ziehen: Dieser Zusammenhang kann deshalb entweder als triviale Feststellung gesehen werden, die keiner näheren Beschäftigung wert ist, ${ }^{6}$ - oder als ein besonders lohnender Forschungsgegenstand. Das Anliegen des gegenwärtigen Bandes entspringt der letzteren Einschätzung. Dafür lassen sich mehrere Gründe anführen: erstens, dass es auf der empirischen Ebene jedenfalls noch viel Neues über die Ausgestaltung dieses Verhältnisses in einzelnen Staaten, Regionen und Zeiten herauszufinden gilt. Lohnend ist diese Erforschung deswegen, weil - zweitens - das Verhältnis von Staat und Raum keineswegs eine Konstante ist, sondern historisch variabel. Es hat sich im Laufe der Zeit in vielfacher Hinsicht entwickelt und verändert, und darüber hinaus ist diese Entwicklung auch nicht in allen Fällen gleich verlaufen. Drittens schließlich sind die beiden Schlüsselkonzepte 'Staat und 'Raum ‘ alles andere als selbstverständlich oder abschließend geklärt. Zu beiden haben in den jüngsten Jahren und Jahrzehnten reichhaltige Debatten in den Sozial- und Kulturwissenschaften Platz gegriffen, in deren Rahmen die in der >Hochmoderne ${ }^{7}$ 
dominierenden Konzeptualisierungen infrage gestellt und neue Theorieangebote vorgebracht worden sind, die jene teils fortführen und erweitern, teils radikal ersetzen sollten. Diese begriffliche Öffnung und Destabilisierung ist zugleich eine Herausforderung und eine Gelegenheit. Das Verhältnis von Staat und Raum kann vor dem Hintergrund dieser Entwicklungen nicht mehr diskutiert werden, ohne dass beide selbst problematisiert werden; die vertiefte Reflexion über sie kann aber auch eine reiche Vielfalt neuer Perspektiven auf das genannte Verhältnis eröffnen, die der empirischen Forschung bisher unbekannte oder ignorierte Fragestellungen, Gegenstände und Quellen erschließen.

Dieser Themenbereich steht einer großen Bandbreite von disziplinären und transdisziplinären Zugängen offen. Wird er jedoch in dieser Zeitschrift behandelt, dann muss speziell die Verwaltung als drittes Glied des Verhältnisses in den Blick treten. Sie ist zwar nicht die einzige, aber dennoch eine sehr wichtige Vermittlungsinstanz zwischen Staat und Raum. Ihre Rolle in dieser Beziehung ist der gemeinsame Gegenstand aller hier versammelten Beiträge - oder vielmehr: Die unterschiedlichen Ausformungen dieser Rolle sind es. Den zeitlichen Rahmen bildet das ılange ‘ 19. Jahrhundert vom Vorabend der Revolutionen am Ende des 18. Jahrhunderts bis zum Ersten Weltkrieg. In diesen Zeitraum fallen nach der überwiegenden Forschungsmeinung entscheidende Entwicklungen im Bereich der Ausweitung und der Verdichtung staatlicher Strukturen im Allgemeinen und staatlicher Verwaltung im Besonderen in weiten Teilen Europas ${ }^{8}$ wie auch der außereuropäischen Welt. ${ }^{9}$ Nach manchen Auffassungen war dies sogar überhaupt jene Epoche, in der Staaten erst ins Leben traten. ${ }^{10}$ Das Urteil darüber hängt davon ab, welche Definition der Staatlichkeit mit welcher Strenge angewendet wird.

Die Artikel dieses Bandes beziehen sich auf größere und kleinere Staaten in Mittel-, West- und Südeuropa: Neben etlichen der damaligen deutschen Fürstentümer sind die Habsburgermonarchie, die Niederlande, Italien, Frankreich und das Osmanische Reich Gegenstand von Einzelstudien. Die Breite der Zusammenstellung und somit - nach unserer Hoffnung - ihr Wert und Reiz für die Leserinnen und Leser liegt in der Diversität der thematischen und methodischen Zugänge innerhalb des Rahmens, der von einer historischen Befassung mit der Dreiheit von Staat, Raum und Verwaltung vorgegeben wird.
Um diese Breite etwas zu strukturieren, sollen in der Einleitung diese drei Komponenten der Reihe nach thematisiert werden. Dabei kann es sich freilich nicht darum handeln, die Geschichte der diesbezüglichen Theoriebildung Revue passieren zu lassen - für den Staat lässt sich die Literatur guten Gewissens als unüberschaubar beschreiben, ${ }^{11}$ für den Raum ist dies inzwischen kaum weniger der Fall, ${ }^{12}$ aber auch für die Verwaltung ist sie zumindest reichlich. Ebenso wenig soll aus dem Spektrum gegenwärtiger Ansätze eine verpflichtende Festlegung auf genau eine Theorie getroffen werden, welche in sämtlichen Beiträgen anzuwenden wäre. Hingegen sollen zunächst einige weit zurückreichende und wirkmächtige Vorstellungen von Staat, Raum und Verwaltung benannt werden, die beim gegenwärtigen Diskussionsstand als fragwürdig, wenn nicht gar als irreführend eingestuft werden müssen - nicht weil ihre Zurückweisung auf theoretischer Ebene irgendeinen Neuigkeitswert beanspruchen könnte, sondern weil sie das Sprechen über diese Konzepte so lange geprägt haben, dass mit ihrem unterschwelligen Fortwirken in wissenschaftlichen Forschungsdesigns und Darstellungen, aber auch in der Alltagssprache weiterhin zu rechnen ist. Ihnen sollen Hinweise auf alternative Blickwinkel entgegengestellt werden, ohne dabei einen Anspruch auf Ausschließlichkeit oder Vollständigkeit zu erheben. Einige dieser neueren Forschungsperspektiven und Gegenstände verbinden jeweils mehrere der hier versammelten Texte. Dies wird insbesondere im vorletzten Abschnitt zum Raum dargelegt.

\section{Vom Leviathan zum stechnostates: Wie kann vom Staat gesprochen werden?}

Eine besonders verbreitete Staatsvorstellung lässt sich an dem vielleicht berühmtesten aller bildlichen Darstellungsversuche beispielhaft erklären: dem 1651 erstmals erschienenen Frontispiz von Thomas Hobbes' "Leviathan«. Die gigantische Figur ist aus vielen winzigen Menschengestalten - es lassen sich über dreihundert zählen - zusammengesetzt, am Übergang vom Rumpf zum Kopf endet jedoch das sichtbare Kompositum und der Staat trägt ein einziges Gesicht, jenes des Königs. ${ }^{13}$ Dieser "homo artificialis" ist also eine einzige Person mit einem Körper und einer Seele, dem Souverän. ${ }^{14}$ Derart 
offene Personifizierungen sind in neueren Theorien freilich nicht anzutreffen, doch ließs und lässt sich immer noch die Angewohnheit der Reifizierung beobachten. Gemeint ist damit die Vorstellung, der Staat sei ein einheitlicher Akteur, dem die Fähigkeit zu handelndem Auftreten zugeschrieben und mitunter sogar ein eigener Wille zugebilligt wird, sei es jener des oder der Individuen an der Staatsspitze oder ein transpersonaler >Gemeinwille`.

"In short «, fasst dies Patrick Joyce zusammen, »the state is pretty much conceived of as a thing. However, the state is not a thing; at least it is not a single active subject «. ${ }^{15}$ In der Tat hält die reifizierte Vorstellung vom Staat keiner empirischen Überprüfung stand, die versucht, den vermeintlichen einheitlichen Willen inhaltlich zu bestimmen, seinen Sitz zu lokalisieren und seine Ausführung zu verifizieren. Dem Staat als Ganzem ist deswegen in vielen neueren Ansätzen mit Recht nicht nur die Handlungsfähigkeit, sondern selbst die behauptete Einheit bestritten worden. ${ }^{16}$ Wenn er sinnvoll gedacht werden kann, dann nur zusammengesetzt aus einer Vielzahl menschlicher, institutioneller und dinglicher Komponenten, die ihm zugeordnet werden oder sich selbst ihm zuordnen, deren Zusammenwirken aber keineswegs immer von Interessenharmonie und von Koordination des Handelns geprägt ist. Wird dies ernst genommen, dann stellen sich selbst alltägliche Gewohnheiten beim Sprechen vom Staat als fragwürdig dar: Im Grunde kann er dann nicht als Subjekt auftreten, dem nachgesagt wird, es habe etwas getan oder gar gewollt. ${ }^{17}$ Vielmehr müsste davon die Rede sein, dass dem Staat zugeordnete Akteure oder Aktanten in seinem Rahmen und in seinem Namen auftreten, sprechen, handeln oder wirken.

Eng mit der Reifizierung des Staates verbunden ist die Vorstellung einer klaren Trennung zwischen ihm und der `Gesellschaft‘, aus der er herausgehoben zu sein, über die er seine Herrschaft und für die er seine Fürsorge auszuüben scheint. ${ }^{18}$ Auch diese Trennungslinie erweist sich bei näherer Untersuchung als schwierig zu lokalisieren, als unbeständig und als in hohem Maße durchlässig. So fragt etwa Michael Mann:

Given such interpenetration, where does the state end and civil society begin? The state [...] contains multiple institutions and tentacles sprawling from the center through its territories, even sometimes through transnational space. Conversely, civil society [sends] out diverse raiding parties - pressure groups and political parties - into the various places of the state, as well as outflanking it transnationally. ${ }^{19}$

Wird die Rede von einer Grenze zwischen Staat und Gesellschaft selbst historisiert, erweist sie sich - so wie auch jene von der wesenhaften Einheit des Staates als kommunikatives Konstrukt mit erkennbaren politischen Intentionen. Manchmal wird von einer solchen Grenze in der Absicht gesprochen, den Einflussbereich des Staates zu limitieren und bestimmte Bereiche von ihm auszunehmen. ${ }^{20}$ In anderen Kontexten lässt sich die Beschreibung des Staates als eine abgegrenzte und herausgehobene eigene Sphäre hingegen als ein Element seiner Legitimierung und Überhöhung verstehen. Die Hervorbringung der Vorstellung einer klaren Grenze und die Verschleierung dieser Hervorbringung durch die Beschwörung vermeintlicher Notwendigkeit oder Selbstverständlichkeit dienen selbst der Sicherung staatlicher Macht, wie Timothy Mitchell festhält:

The line between state and society is not the perimeter of an intrinsic entity, which can be thought of as a free-standing object or actor. It is a line drawn internally, within the network of institutional mechanisms through which a certain social and political order is maintained. [...] But this does not mean the line is illusory. On the contrary, [...] producing and maintaining the distinction between state and society is itself a mechanism that generates resources of power. ${ }^{21}$

Hinzuzufügen wäre, dass die Linie zwischen staatlichem und nichtstaatlichem Bereich oftmals nicht zwischen einzelnen menschlichen und institutionellen Akteuren gezogen wird, sondern gleichsam in deren Innerem, denn die Zuordnung zum Staat ist bei Institutionen nicht immer und bei Menschen nie eine exklusive, die jegliche anderen Zugehörigkeiten und Bindungen ausschaltet auch wenn staatliche Institutionen von ihren Agenten und Agentinnen meist bevorrangte Loyalität einfordern. Gerade Angehörige lokaler Eliten nahmen und nehmen oft zwiespältige Stellungen ein, in denen sich die Vertretung des Staates gegenüber lokalen Gemeinschaften und dieser Gemeinschaften gegenüber höheren staatlichen Stellen, die Ausübung vom Staat übertragener Befugnisse und die Nutzung und Vermehrung autochthoner Macht 
miteinander verbinden. ${ }^{22}$ Dies zeigen in unterschiedlichsten Zusammenhängen die Beiträge von Josef Löffler für vormärzliche Grundherrschaften in Österreich, Böhmen und Mähren, von Anette Schlimm für Dorfbürgermeister in Preußen und Bayern sowie von Rüdiger von Krosigk für die ehrenamtlichen Bezirksräte in Baden. Mit den Bürgermeistern als Verbindungsgliedern zwischen Staat und Dorf befasst sich auch die Relektüre von Bettina Severin-Barboutie zu einer bis heute unersetzten sozialgeschichtlichen Großstudie aus den 1980er Jahren unter der Leitung von Maurice Agulhon.

All dies bleibt nicht ohne Auswirkung auf die Frage nach den Triebkräften und Hauptakteuren des Staatsausbaus. Wird der Staat als handlungsfähiges Subjekt oder als abgetrennte Sphäre vorgestellt, geht dies meist mit der Auffassung einher, dass er selbst, einmal ins Leben getreten, der ausschließliche oder zumindest der wichtigste Betreiber seiner eigenen Expansion sei. Die Tendenz dazu erscheint als in seinem Wesen notwendig angelegt; zugleich wird wachsende Durchstaatlichung ${ }^{23}$ häufig nicht nur als auffallendes Phänomen, sondern als eine nachgerade geschichtsnotwendige Komponente einer allgemeinen Modernisierung und Rationalisierung in erster Linie der europäisch-atlantischen Gesellschaften gedeutet. Solche Gedankengänge finden sich voll ausformuliert bei Max Weber, ${ }^{24}$ in wichtigen Zügen aber auch schon ein gutes halbes Jahrhundert vor ihm bei Alexis de Tocqueville. ${ }^{25}$

Dass dem Staat zugeordnete Akteure oft, und nicht selten absichtsvoll planend, im Sinne einer Erweiterung der Tätigkeiten, Kompetenzen und Machtmittel des Staates gewirkt haben, steht außer Frage. Doch haben neuere Forschungen vor allem zur Frühen Neuzeit die Linearität und Ausschließlichkeit diesbezüglicher Narrative in mehrerlei Hinsicht zu relativieren vermocht. Zunächst haben sie darauf aufmerksam gemacht, dass die Expansion nicht kontinuierlich verlief, sondern in allen entstehenden Staaten auch Phasen ihrer Stagnation, ja mitunter ihrer Rückläufigkeit festzustellen sind. ${ }^{26}$ Schon die Aufrechterhaltung des erreichten Standes zeigte sich als keine unerhebliche Aufgabe für die Staaten sowie deren Agenten und Agentinnen. Darüber hinaus wurde in vielen Einzelstudien gezeigt, dass die Träger und Trägerinnen staatlicher Macht nicht die einzigen waren, deren Rolle im Prozess der Staatsbildung berücksichtigt werden muss. ${ }^{27}$ Vielmehr wirkten auf diesen auch »communities, corporate entities, interest groups and subjects from local society « ${ }^{28}$ durch die Artikulation ihrer Interessen und Wertvorstellungen fortwährend ein. Nicht nur setzten sie sich mit unterschiedlichen Mitteln gegen Zugriffe zur Wehr, die sie als ungerechtfertigt oder allzu belastend empfanden, sondern sie begehrten von den Herrschenden auch ausdrücklich Leistungen der Verwaltung und Justiz, nutzten jene zu vielfältigen eigenen Zwecken, ${ }^{29}$ speisten im eigenen Interesse Information ${ }^{30}$ und andere Ressourcen ein, beteiligten sich aktiv an der Ausübung staatlicher Herrschaft oder versuchten selbst Herrschaft auszuüben oder gar zu konstituieren - mit dem Staat und in ihm, neben ihm oder auch gegen ihn. Die lokale Durchsetzung und der alltägliche Vollzug staatlicher Herrschaft erfolgten also "nicht nur durch Zwang nach dem Schema Befehl und Gehorsam [...], sondern in komplexen Kommunikations- und Interaktionsprozessen «, ${ }^{31}$ welche in der deutschsprachigen Forschung oft mit dem Begriff "Aushandeln «, ${ }^{2}$ auf Englisch etwa mit "persuasion « oder "negotiation « umschrieben werden. ${ }^{33}$ Damit soll nicht, wie gelegentlich kritisiert worden ist, ${ }^{34}$ der Macht- und oft auch Unterdrückungsaspekt ausgeblendet werden - oder gar die vertragstheoretische Fiktion hingenommen, nach welcher staatliche Herrschaft durch ihre vermeintliche Einsetzung seitens der Beherrschten und die daraus erfließende Identität von Staatsgewalt und Staatsvolk legitimiert sei..$^{35}$ Dass die Interaktionen, in denen sich Herrschaft - lokal wie auch überlokal konstituiert und vollzieht, überwiegend asymmetrisch sind, braucht nicht geleugnet zu werden, um in den Blick zu bekommen, dass sie nicht unidirektional sind.$^{36}$ Einen Fokus auf die ,von unten ‘ kommenden Komponenten der Staatsausbauprozesse legen in diesem Band die Texte von Anette Schlimm sowie von Thomas Stockinger über die erstmalige Einrichtung einer staatlichen Bezirksverwaltung in der Habsburgermonarchie.

Eine solche interaktionszentrierte Perspektive unterläuft auch die dem Weber'schen Narrativ innewohnende Teleologie, die Unterstellung von Unumgänglichkeit oder gar Intentionalität, »als hätten die Staatsmänner der Zeit das Ziel gehabt, einen modernen Staat zu schaffen und als hätten sie ihr politisches Handeln auf dieses Ziel hin abgestimmt «. ${ }^{37}$ Vielmehr erscheinen Staatsausbau wie auch gelegentlicher Staatsrückbau als kumulative Auswirkung zahlreicher situativ geprägter Einzelentscheidungen und Handlungsweisen, als »unintended outcome 
of interactive processes «, ${ }^{38}$ deren expansive Tendenz möglicherweise in der inneren Logik von bestimmten Praktiken angelegt, aber den Ausübenden nicht unbedingt bewusst oder gar ein besonderes Anliegen war. Ellinor Forsters Beitrag bietet hierfür ein Beispiel, indem gezeigt wird, dass die Entflechtung von Herrschaftsräumen im Tiroler Zillertal nicht erst begann, als die habsburgischen und die salzburgischen Zentralbehörden ein Interesse an der Arrondierung ihres jeweiligen Territoriums entwickelten, sondern bereits Jahrzehnte zuvor als Taktik in Auseinandersetzungen zwischen ihren lokalen Vertretern aufkam: ein später klassisches Merkmal 'moderner Staatlichkeit als emergentes Resultat ganz anders motivierten Agierens.

Wenn der Staat kein handelndes Subjekt, keine in sich abgeschlossene Sphäre und auch kein ausschließliches Projekt derer ist, die in ihm Machtstellungen innehaben, wie kann er dann beschrieben werden? Wie bereits angedeutet, bietet es sich jedenfalls an, ihn dezentral oder wenigstens polyzentrisch $\mathrm{zu}$ betrachten. $\mathrm{Zu}$ den vielen Menschen, die ihm in unterschiedlichen Graden und Weisen zugeordnet sind, sind Korporationen und Institutionen als Bausteine des Staates hinzuzunehmen; im Sinne des ımaterial turn oder der Actor-Network-Theory nach Bruno Latour kann dies auch auf dingliche Aktanten erweitert werden. Der Staat kann aber nicht als bloße Anhäufung aller dieser Bestandteile gedacht werden, es müssen auch die Verbindungen zwischen ihnen beschrieben werden. In diesem Sinne umschreibt Patrick Joyce ihn als eine Anordnung: "something like an sassemblage`, which is held together (sometimes very uncertainly) at particular key sites or nodes and through the actions of key actors and processes, human and non-human «. ${ }^{39} \mathrm{Zu}$ all dem, was sie umgibt, ihr aber nicht angehören soll, steht diese Anordnung in einem Spannungsverhältnis, das sowohl von dem Streben nach Singularisierung als auch von unhintergehbarer Eingebundenheit geprägt ist, was sich in Unschärfe und oftmaliger Verschiebung der behaupteten Grenzen äußert.

Assemblagen sind aus heterogenen Teilen zusammengesetzte Gesamtheiten, deren Eigenschaften aus den Interaktionen jener Teile resultieren. ${ }^{40}$ Eine der wesentlichen Dimensionen derartiger Ensembles stellen Prozesse der Territorialisierung und der Deterritorialisierung dar, je nachdem, ob Komponenten zusammenwachsen, somit die Identität einer Assemblage stabilisieren und ihre innere
Homogenität vergrößern respektive Grenzen schärfen oder aber manche ihrer Teile auf die Assemblage destabilisierend, also deterritorialisierend wirken. ${ }^{41}$ So stellt sich etwa die Frage, wie aus Provinzen Staaten werden, was der Artikel von Luigi Blanco am Beispiel der Einigung Italiens untersucht. Anna Gianna Manca wiederum zeigt am Beispiel Preußens, dass bei der Formierung neuer Provinzen 1815 vor allem historisch-feudale Gesichtspunkte tragend waren und nicht die von der Verwaltung favorisierten geographischen und wirtschaftlichen Kriterien, welche die Bildung einer gesamtstaatlichen preuSischen Perspektive avisierten. Um 1848 versuchten die Liberalen - erneut erfolglos - preußisches Nationalgefühl zu stärken, indem sie die aus ihrer Sicht überflüssige Mittelinstanz der Provinzen aufheben und nur die Verwaltung in Gemeinden und Kreisen fortbestehen lassen wollten. Paradoxerweise stellen sich dabei die antikonstitutionellen, konservativ-feudalen preußischen Provinzen letztlich als territorialisierend dar: Sie blieben die wichtigste Territorialeinteilung Preußens bis zum Ende des Staates 1947. Analog und doch völlig verschieden gestaltete sich eine sprovinzielle Staatsbildung in den Niederlanden. Stefan Couperus, Harm Kaal, Nico Randeraad und Paul van Trigt zeigen in ihrem Beitrag am Beispiel Südhollands, wie sich Provinzialverwaltungen an der Staatsbildung beteiligten, indem sie sich als administrative Institutionen des neuen Einheitsstaates erfanden und mittels slittle tools of knowledge ${ }^{42}$ und Infrastrukturprojekten die Provinz als integrativen Raum eigenen Rechts hervorbrachten.

Die Gemeinsamkeiten oder Verbindungen zwischen den einzelnen Komponenten des Staates können auf unterschiedliche Weisen perspektiviert werden. Sie können in gemeinsamen Zwecken oder Zielen gesehen werden; allerdings sind die erklärten und tatsächlich verfolgten Ziele von Staaten so vielfältig, dass schon Max Weber eine Definition über den Zweck für unmöglich befunden hat. ${ }^{43}$ Doch ist das von Weber als Definitionsmerkmal gebrauchte Machtmittel, das Monopol legitimer Gewaltanwendung, angesichts des enormen Aufwands zu seiner Durchsetzung und Erhaltung, die auch stets nur sehr unvollkommen gelingt, ${ }^{44}$ selbst als Ziel zu verstehen. Der Staat ist in dieser Hinsicht eine Anordnung von Akteuren und Prozessen, deren Wirken dazu tendiert, bestimmte Formen von Machtausübung - nicht nur physische Gewalt, auch wenn sie mit Weber weiterhin als besonderes 
Signum und sultima ratio ‘ des Staates gelten kann ${ }^{45}$ - innerhalb eines festgelegten Geltungsbereichs für sich selbst zu monopolisieren und ihren Gebrauch durch andere als illegitim zu brandmarken. Gerade zur Operationalisierung eines solchen Monopols bedarf es im Übrigen der Grenzen, die zwischen Staatlichem und Nichtstaatlichem gezogen werden. Eng verwandt ist die von Pierre Bourdieu angebotene Beschreibung, wonach die wesentliche Tätigkeit des Staates die Konzentration verschiedener Arten von >Kapitalien s sei, wozu er die körperliche Gewalt, aber auch etwa ökonomisches Kapital, Information oder kulturelles Kapital rechnet. ${ }^{46}$

Der Zusammenhalt eines polyzentrischen Staates kann in institutionellen Strukturen gesehen werden, beispielsweise in der Festlegung eines politologischen Handbuchs darauf, »den Staat als einen institutionalisierten Handlungskontext « anzuschauen, »in dem Individuen, Gruppen (kollektive Akteure) oder Organisationen (korporative Akteure) zusammenwirken [...], um bestimmte gesellschaftliche Probleme zu lösen bzw. Aufgaben zu erfüllen «. ${ }^{47}$ Mit Institutionen können dabei Organisationen, aber auch Normen und Verfahren gemeint sein. Dennoch schließt der Bereich des Institutionalisierten, auch wenn er sehr weit gefasst wird, sicherlich nur einen Teil dessen ein, was den Staat zusammenhält. Es ist eine ebenfalls nicht mehr neue Erkenntnis, dass die Wirksamkeit des Staates abhängig ist von seiner Akzeptanz, ja vom überwiegenden Glauben der ihm Angehörigen und Unterworfenen an seine legitime Existenz:

[The] recognition of the state as a significant factor in political and social life [...] depends [...] on the existence of a cultural disposition to allot recognition to the conceptual existence of a state at all. [...] To what extent have individuals generalized the concept and cognition of state in their perceptions and actions, and to what extent are such cognitions salient? ${ }^{48}$

In dieser Sicht beruht der Bestand des Staates auf vielfältigen, miteinander zusammenhängenden Bedeutungszuweisungen: Zuschreibungen von Eigenschaften und Handlungsmöglichkeiten an den Staat, Zuordnungen von Akteuren und Ressourcen zum Staat. Erzeugt, verbreitet und fortwährend aktualisiert werden diese Zuweisungen zunächst mehr oder minder explizit durch Sprechakte, die sich zu Diskursen verbinden; zugleich in Praktiken und Handlungsweisen, die den Staat realisieren - in dem Sinne, dass sie ihn wahrnehmbar machen, ihn dem Erleben und der Erfahrung der Menschen einschreiben. Die Verdichtung dieser Diskurse und Praktiken hat als langfristige, teilweise intendierte und teilweise emergente Auswirkung gezeitigt, dass die Existenz des Staates nicht bloß akzeptiert, sondern für die meisten Menschen selbstverständlich wurde. ${ }^{49}$

Nicht zu vernachlässigen ist außerdem, welche Rolle materielle Objekte für den Zusammenhalt und das Funktionieren des Staates spielen. "Capacities for action, including action upon humans, are built into objects so to speak, and outcomes shaped by them «, ${ }^{50}$ so lässt sich die Grundannahme des smaterial turn k kurz umschreiben. Unter diesem Blickwinkel waren bestimmte Technologien und ihre Integration zu technologischen Systemen ${ }^{51}$ für den Aufbau des Staates mitbestimmende Faktoren, deren Auswirkungen sich mit den Intentionen von Menschen bei ihrer Erzeugung und Nutzung nur teilweise erklären lassen. Jene unbeabsichtigten Konsequenzen, die bereits oben als nicht zu vernachlässigendes Moment bei der Entstehung staatlicher Strukturen angesprochen wurden, können sich demnach nicht nur aus menschlichen Verfahren und Verhaltensdispositionen, sondern auch aus den Eigenschaften von Objekten und technischen Lösungen ergeben. ${ }^{52}$ Im Hinblick auf den Staat und die Verwaltung des 19. Jahrhunderts kommen dabei etwa die Kommunikations- und Transporttechnologien in Betracht, die in jener Zeit eine rasante Weiterentwicklung erfuhren und kaum zu überschätzende Beiträge zu den Wandlungen in Wirtschaft und Gesellschaft leisteten. ${ }^{53}$ Insbesondere der Eisenbahn, welche im Aufsatz von Nadja Weck thematisiert wird, kam in diesem Zusammenhang eine tragende Rolle zu. Gegenstand großer staatlicher Anstrengungen und Instrument vertiefter Einwirkung auf Territorium und Bevölkerung waren auch Interventionen in die Landschaft wie die Meliorationen und Wasserbauprojekte, ${ }^{54}$ deren Rolle im Artikel von Stefan Couperus, Harm Kaal, Nico Randeraad und Paul van Trigt zur Darstellung gelangt. Kaum zu überschätzen sind in ihrer Wichtigkeit zudem die Techniken der Speicherung und Verarbeitung von Information. Ihr Ausbau im 19. Jahrhundert war gleichfalls von größter Bedeutung für Staat und Verwaltung, ${ }^{55}$ obwohl er vorerst weniger auf bahnbrechenden neuen Erfindungen beruhte als auf der Erweiterung und Verfeinerung der Möglichkeiten, die von 
zwei bereits sehr alten und lange schon im Dienst von Herrschaft stehenden Technologien geboten wurden: der Schrift ${ }^{56}$ und dem Papier, ${ }^{57}$ wie auch in diesem Band die Beiträge von Rüdiger von Krosigk und von Birgit Näther demonstrieren. Insofern der Staat gerade in dem Zeitraum, den die hier versammelten Artikel behandeln, mehr denn je existenziell auf die Leistungen dieser und weiterer technologischer Systeme angewiesen war, lässt sich seine Entwicklung während dieser Zeit als »coming of the technostate umschreiben. $^{58}$

\section{Von der Verwaltung zum Verwalten}

Eng verknüpft mit der analytischen Öffnung und theoretischen Rekonzeptualisierung des Staates ist auch eine kulturwissenschaftlich informierte Auseinandersetzung mit der Verwaltung. Der moderne Begriff der >Verwaltung ist in politischen, staatsrechtlichen und staatswissenschaftlichen Schriften des frühen 19. Jahrhunderts entworfen worden. Das Substantiv >Verwaltung، gibt es im Deutschen zwar seit dem 15. Jahrhundert, aber erst im 18. Jahrhundert ist der "Aktionsbegriff « auch zu einem "Institutionsbegriff « geworden, haben »die Verwaltungstätigkeiten sich selber so weit spezifiziert und auseinanderentfaltet [...], daß sie einer übergreifenden Systematik bedurften ${ }^{59}$ Dabei haben sich die Verwaltungspraktiken nicht nur "selber « spezifiziert und ausdifferenziert, sondern ihre Systematisierung selbst verdankt sich administrativen Praktiken. Jedenfalls sind die Traktate, welche den neuen Begriff der Verwaltung formieren, ausnahmslos von Verwaltungspraktikern, das heißt von Sekretären oder Beamten, verfasst worden. Insofern ist die diskursive Formation der Verwaltung selbst Effekt administrativer Schreibakte.

Die Systematik des Verwaltungsbegriffs ist im Zeitalter von Konstitutionalismus und liberal-bürgerlichen Revolutionen aus der staatswissenschaftlichen Differenzierung und Eingrenzung der absolutistischen Universalbegriffe >Policey` und >Regierung`hervorgegangen. Erste Bedingung der Möglichkeit für diese Serie von Differenzierungen und das Hervortreten der >Verwaltung، ist die Auflösung der absolutistischen `Policey`, die der Kameral- und Policeywissenschaftler Johann Heinrich Gottlob von Justi noch als "alle Maaßregeln in innerlichen
Landesangelegenheiten « definiert hatte, "wodurch das allgemeine Vermögen des Staats dauerhaftiger gegründet und vermehret, die Kräfte des Staats besser gebrauchet und überhaupt die Glückseligkeit des gemeinen Wesens befördert werden kann «. ${ }^{60}$ Indem die moderne >Polizei` auf Sicherheit, Gefahrenabwehr und Schadensverhütung verwiesen wird und einen rein negativen Sinn annimmt, ${ }^{61}$ verwaist ein ganzer Komplex staatlicher Tätigkeit. Mit der Redefinition der >Policey` entsteht ein leeres und zugleich juridisch wie politisch abgegrenztes Feld.

Zweite Bedingung der Möglichkeit für das Hervortreten der >Verwaltung ‘ ist Montesquieus Lehre der Gewaltenteilung. In "De l'esprit des lois« postulierte der französische Staatstheoretiker der Aufklärung drei Arten der Staatsgewalt: eine legislative, gesetzgebende Gewalt, eine exekutive, vollziehende Gewalt und eine judikative, richterliche Gewalt. ${ }^{62}$ Montesquieu nannte die vollziehende Gewalt auch >Verwaltung`, allerdings ohne diesen Begriff genauer zu bestimmen. ${ }^{63}$ Der weitgefasste und unspezifische Terminus , Verwaltung « machte im 19. Jahrhundert sowohl eine »Bedeutungserweiterung als auch eine Bedeutungsverengung durch, indem [er] jetzt einerseits auch auf den Organisationstatbestand der Verwaltung angewandt und andererseits endgültig auf Vollzugsfunktionen beschränkt" wurde. ${ }^{64}$ Diese Auffassung von >Verwaltung، gilt bis weit ins 20. Jahrhundert, auf jeden Fall ist sie charakteristisch für das lange 19. Jahrhundert. Damit ist in aller Regel allein die öffentliche Verwaltung gemeint, ohne dass dies explizit gesagt $\mathrm{zu}$ werden bräuchte.

Sind es Beamte und Sekretäre gewesen, welche die >Verwaltung a auf ihren Begriff brachten und instituierten, waren es zunächst Juristen und Ende des 19. Jahrhunderts juristisch geschulte Sozialwissenschaftler in Deutschland, Frankreich und den USA, welche die gelehrte Reflexion dieser Verwaltung initiierten. ${ }^{65}$ Die Verwaltungswissenschaft, die aus der Emanzipation der Verwaltungsrechtswissenschaft des 19. Jahrhunderts hervorging, konzipiert Verwaltung als Komplement der Regierung. Im Unterschied zur Regierung ist ihre Aufgabe nicht die Setzung politischer Programme, sondern deren Umsetzung. ${ }^{66}$ Ende des 19. und bis weit ins 20. Jahrhundert hinein ist Verwaltungsgeschichte vorwiegend als Verfassungsgeschichte betrieben worden. Diese in erster Linie juristisch orientierten Studien interessierten sich vor allem für Institute und Institutionen, werteten 
vorab normative Quellen wie Gesetze und Verordnungen aus. Verwaltung stellte sich in diesem Kontext primär als normativer Vollzug von Politik und Recht dar. ${ }^{67}$ Demgegenüber interessierte sich die Soziologie in der ersten Hälfte des 20. Jahrhunderts nach den posthum veröffentlichten Studien von Max Weber weniger für Verwaltung als vielmehr für Bürokratie, die er als rationallegale Herrschaft definierte. ${ }^{68}$ Es waren schließlich Überlegungen Webers, die im Umkreis der Bielefelder Schule in den 1960er und 1970er Jahren der Geschichte der Verwaltung neue Impulse gaben. ${ }^{69}$ Diese sozialhistorischen Untersuchungen der Verwaltung rückten im Unterschied zu den älteren verfassungsgeschichtlichen Ansätzen eher Strukturen sowie Angestellte und Beamte als soziale Gruppe ins Zentrum des Interesses.

Seit den 1990er Jahren destabilisiert eine Reihe von Prozessen die grundlegenden Rahmenbedingungen, unter denen öffentliche Verwaltung arbeitet und ihr Selbstverständnis herausbildet. Mit der Entgrenzung zwischen öffentlichem und privatem Sektor, der Ökonomisierung, Transnationalisierung, Digitalisierung und Vernetzung schwand auch das Leitbild der modernen Bürokratie als in sich geschlossener, hierarchisch gegliederter Apparat mit klaren Kompetenzen und Zuständigkeiten. Stattdessen etablierten sich neue Konzepte wie 'governance und >New Public Management $\triangleleft .{ }^{70}$ Im Zuge dieser Transformation erfuhr die wissenschaftliche Erforschung der Verwaltung eine analytische Reperspektivierung ähnlich jener des Staats. An die Stelle der selbstredend öffentlichen Verwaltung trat vermehrt die wissenschaftliche Untersuchung uneinheitlicher, hybrider administrativer Gebilde. ${ }^{71}$ Dieser Verschiebung ging zuerst ein gesteigertes methodisches Interesse für Materialitäten (`paperwork`) und schließlich für Praktiken der Verwaltung voraus. ${ }^{72}$ Anschließsend an Studien von Niklas Luhmann, Cornelia Vismann ${ }^{73}$ und Bruno Latour konzeptualisieren deutsche Medienwissenschaftlerinnen und Medienwissenschaftler Verwaltung als Kulturtechnik. ${ }^{74}$

Die Kulturtechnikforschung stellt nicht mehr die Institution, die Verwaltung, in den Mittelpunkt, sondern die Praxis: das Verwalten. Bei den Tätigkeiten des Verwaltens anzusetzen heißt nicht, die institutionellen, ökonomischen, politischen und kulturellen Kontexte zu ignorieren, sondern diese dort zu berücksichtigen, wo Angestellte, Beamte und Beamtinnen sie tagtäglich erleben, produzieren und reproduzieren. Wie, unter welchen
Bedingungen und in welchen Arbeitsumgebungen erzeugen und bearbeiten sie zum Beispiel Verwaltungsschriftgut? Welche Rolle spielen Akten »in diesem Wirklichen, von dem sie sprechen «? ${ }^{75}$ Aus dieser Perspektive werden Akten weniger auf ihre Inhalte befragt, "als Zeugnisse einer wie auch immer gearteten historischen Realität«, sondern vielmehr »als Zeugnisse der durch sie selbst verkörperten Praktiken und Kulturtechniken « analysiert. ${ }^{76}$ Administrative Praktiken und Arbeitsumgebungen als Kulturtechnik zu beschreiben, heißt das »komplexe[...] Akteur-Netzwerk « rekonstruieren, »das technische Objekte und die Handlungsketten einbegreift, in die sie eingebunden sind, die sie konfigurieren oder die sie konstitutiv hervorbringen $"{ }^{77}$ Akten erweisen sich dabei als Kulturtechniken des Entscheidens.

Nach Dirk Baecker ist eine Entscheidung eine Kommunikation, "die an andere Kommunikationen adressiert wird (so, wie die eine Akte auf eine andere Akte verweist und jeder Akt einen anderen Akt fordert und abweist zugleich), um weitere Entscheidungen zu ermöglichen “. ${ }^{78}$ Akten bestehen aus Schreiben und deren Beilagen, die ein Antwortschreiben verlangen, dem wiederum alle vorgehenden Schreiben zu einem Geschäft beiliegen. Adressaten greifen darin Inhalte und Vorschläge von Absendern oder Beilagen auf und verleihen dadurch jenen Vorschlägen ein stärkeres Gewicht. Der Bezug auf Kommunikationsangebote vorheriger Akten fabriziert eine Entscheidung, ohne dass diese vor der Bezugnahme schon feststehen würde. ${ }^{79}$ Akten konstituieren Verfahren: Sie verleiten zur Handlung der Bezugnahme auf vorliegende Schreiben und bahnen so eine Entscheidung an. Das »erklärt, weshalb etwas Macht an einen durchschnittlichen Geist abgegeben wird, einfach indem man Akten durchsieht «. ${ }^{80}$ Akten statten Akteure mit limitierter Handlungsmacht aus, begrenzen Assoziationen, bringen mit anderen Worten Beamte hervor. Zugleich assoziieren Akten Beamte und Vorgesetzte, Administrationen und Subjekte und erzeugen so auf Wiederholung basierende bürokratische Strukturen. ${ }^{81}$

Eine solche auf Praxis und Kulturtechnik fokussierte Analyse evoziert die Öffnung des untersuchten Forschungsgegenstandes. Neben öffentlichen, rechtlichen und staatlich instituierten Verwaltungsakten interessieren auch haushälterisch, kirchlich, literarisch, privat- oder marktwirtschaftlich, suprastaatlich, zivilgesellschaftlich organisierte Verwaltungspraktiken. ${ }^{82}$ Dabei verflüssigt 
sich der im 18. Jahrhundert etablierte und im 19. Jahrhundert vergegenständlichte Kollektivsingular ,Verwaltung`. Verwalten mutiert zu einem weitverbreiteten und vielfältigen Register des Handelns, das nicht zwangsläufig an staatliche oder überhaupt an Formen der Herrschaft gekoppelt ist. Öffentliche Verwaltung im Allgemeinen und Bürokratie im Besonderen stellen dann nicht mehr den Proto- oder Idealtypus der Verwaltung dar, sondern erscheinen vielmehr als ihre erklärungsbedürftigen Spezialfälle ${ }^{83}$ - Assemblagen, zusammengesetzt aus kontingenten, unscheinbaren Akten. Damit öffnet sich für die Geschichte ein nichtlineares Feld, in dem Verwaltung nicht mehr teleologisch modernistischer Bewertung unterzogen, sondern nach ihrem Eigenwert und ihrer Eigenlogik bemessen werden kann: die Austreibung des Staates aus der Verwaltung. ${ }^{84}$

Eine solche Genealogie skizzierte Michel Foucault in seinen Vorlesungen zur Geschichte der Gouvernementalität. Darin wird herausgearbeitet, wie im 18. Jahrhundert eine neue Regierungskunst entsteht. 'Regierung‘, 'Regiment bezeichnete bis ins 18. Jahrhundert die gesamte Staatstätigkeit, "die Verwaltung des gemeinen Wesens und Besorgung alles dessen, so zur Erhaltung und Beförderung der Wohlfahrt des Staates erspriesslich ist ${ }^{85}$ Gouvernementalität, die neue Regierungskunst, formiert sich exakt an der Stelle, wo die >Policey` des Ancien Régime, diese überreglementierende Regierungskunst, auseinanderfällt. Das Auftauchen des Liberalismus, die Freiheit als unverzichtbarer Bestandteil der Gouvernementalität, schafft die Grundlagen einer Verwaltung, die das notwendige Komplement zur politischen Ökonomie und ihren quasinatürlichen Mechanismen der Selbstregulierung darstellt:

\begin{abstract}
Man wird beeinflussen, anreizen, erleichtern, tun lassen müssen: Mit anderen Worten, man wird verwalten, und nicht mehr reglementieren müssen. Diese Verwaltung wird als wesentliches Ziel nicht sosehr die Verhinderung von Dingen haben, sondern es so einzurichten, daß die notwendigen und natürlichen Regulationen greifen können, oder auch Regulierungen festzulegen, die die natürliche Regulation gestatten werden. ${ }^{86}$
\end{abstract}

Verwalten heißt dann nicht mehr reglementieren, sondern regulieren - ökonomisch statt policeylich handeln.
Damit die Kräfte des freien Marktes und der Nationen sich entfalten können, müssen sie in Gang gebracht und gehalten werden. Letzteres ist nach Foucaults Geschichte der Gouvernementalität die paradoxe Aufgabe der Verwaltung: natürliche Regulationen zu ermöglichen.

Derart separiert und in Anschlag gebracht, vermag die Verwaltung oder vielmehr die Tätigkeit des Verwaltens neue Perspektiven auf Raum und Staat aufzuwerfen. Wenn Verwaltung nicht mehr a priori staatliches Handeln abzüglich Rechtsprechung und Gesetzgebung ist, ${ }^{87}$ stellt sich umgekehrt die Frage, welche Akte Handeln staatlich machen. Bezogen auf den Raum lautet die Frage: Mithilfe welcher Praktiken und Techniken konstruierten Beamte und Sekretäre welche - staatlichen oder anderen - Räume, und in welcher Ausdehnung? Und umgekehrt: Was für Räume benötigte die Verwaltung oder die Tätigkeit des Verwaltens? Was für Räume schaffen administrative Praktiken für die Verwaltung selbst?

\section{Physische und virtuelle Räume, ihre Konstruktion und Nutzung}

Die Rede vom Raum als Begriff und Metapher ist in der Gegenwart sehr verbreitet. ${ }^{88}$ Werden hier auch die kaum zu überschauenden Spielarten räumlicher Metaphern für Zusammenhänge des menschlichen Lebens hinzugezählt, wozu so banale Redeweisen wie die Umschreibung sozialer Hierarchien mit den Worten >oben` und > unten ' gehören, ${ }^{89}$ dann kann sie sogar als geradezu ubiquitär bezeichnet werden. Sie ist zudem sehr alt, in ihren historischen Varianten aber auch einer Vielzahl von Moden und Weiterentwicklungen unterworfen, die einen sich verändernden Fundus an Raumvorstellungen indizieren - als eher zufällige Kette von Beispielen seien genannt: die Gewohnheit frühneuzeitlicher Gelehrter, bibliographische Nachschlagewerke mit `Bibliotheca ${ }^{90}$ und Darstellungen mit >Theatrum ‘ oder `Schau-Platz $` \mathrm{zu}$ betiteln; ${ }^{91}$ der von dem Sozialgeographen Friedrich Ratzel aufgebrachte und später vom Nationalsozialismus vereinnahmte >Lebensraum`-Begriff in der Anwendung auf menschliche Gruppen; ${ }^{92}$ schließlich noch die heute in der Wissenschaft wie der Alltagssprache teils mit Erkenntnisgewinn eingesetzte, teils bis zur Sinnentleerung strapazierte Rede von `Netzwerken`. 
Diese Verwendungen von Raumvorstellungen finden auf unterschiedlichen epistemologischen Ebenen statt respektive kombinieren diese vielfältig miteinander. ${ }^{93}$ Oft beruhen sie indessen auf wenig reflektierten Vorstellungen davon, was mit >Raum ‘ überhaupt gemeint ist oder sein kann. Vor allem setzen sie meist implizit oder sogar explizit voraus, dass `Raum` jeder menschlichen Wahrnehmung und Nutzung präexistent ist, "eine Art physisches Apriori «, ${ }^{44}$ »eine (empirisch) faßbare Entität, [die] als gegebene Substanz, mehr oder weniger unabhängig von ihrer Erfassung, vorgestellt werden kann «. ${ }^{95}$ In den Sozial- und Kulturwissenschaften führt eine solche Sicht dazu, dass Raum entweder als "nicht weiter bemerkenswert « außer Acht gelassen wird ${ }^{96}$ oder ausschließlich als erklärender Faktor für auf ihn bezogene menschliche Phänomene in den Blick gelangt. ${ }^{97}$ Diese Anschauungen sind in den vergangenen Jahrzehnten im Zuge einer oft als spatial turn wegung in den Sozial- und Kulturwissenschaften vielfach kritisiert worden. ${ }^{98}$ Ihnen wird die These entgegengesetzt, dass Räume sozial und kulturell konstituiert werden. Die Vorgänge dieser Konstitution können als soziale Interaktion oder als Kommunikation aufgefasst werden. ${ }^{99}$ Dabei tritt die Vorstellung eines rein physikalisch oder mathematisch bestimmbaren Raums in den Hintergrund, ohne doch ganz verworfen werden zu können; ${ }^{100}$ sie erscheint aber nur als eine Komponente, die in die Konstitution von Räumen einfließst. ${ }^{101}$ So ist es zwar möglich, zwischen Räumen als "physisch-materielle Phänomene«, als »hybride Phänomene, in denen sich Physisches und Soziales mischt", und schließlich als "ausschließlich sozial konstituierte Sinnphänomene « zu unterscheiden. ${ }^{102}$ Sobald es aber um das Verhältnis von Menschen zu Räumen geht, sind nur noch die beiden letzteren Fälle relevant, da jeder physische Raum unweigerlich zum hybriden Phänomen wird, sobald ihn Menschen wahrnehmen, beschreiben, betreten, sich in ihm aufhalten oder bewegen oder in welcher Weise auch immer mit ihm in Verbindung treten. Zudem ist die Grenzziehung zwischen einem physikalisch bestimmbaren und einem sozialen Raum stets auch ein politischer Akt, indem sie manche Raumeigenschaften als vorgegeben, andere als gemacht und somit disponibel einstuft. ${ }^{103}$ "Erfassung und Konstruktion eines Raums [...] sind nicht trennscharf abgrenzbar «, ${ }^{104}$ und alle Räume sind deswegen zumindest teilweise virtuell.
Um dies an einem anschaulichen Beispiel zu illustrieren: Die physische Existenz der Berge, die heute 'die Alpen genannt werden, geht unbestreitbar der Präsenz von Menschen in ihrer Nähe weit voraus. Allerdings liegt es an von Menschen gesetzten Bedeutungszuweisungen und Handlungen, ob diese Berge nun als ein zusammenhängendes Gebirge mit dem Namen >die Alpen ‘ betrachtet werden, welche Teilmengen ihrer Felsmasse als benennbare Bergketten und als einzelne Berge abgegrenzt werden, ob sie als bedrohliche Wildnis, als der monastischen Einsamkeit zuträgliche Einöde, als zu überwindendes Verkehrshindernis, als Lager wertvoller Rohstoffe wie Holz oder Mineralien, als militärisch zu verteidigende Festung, als pittoreske Touristenattraktion wahrgenommen und behandelt werden. Diese Arten des Umgangs führen zudem über menschliche Interventionen auch $\mathrm{zu}$ jeweils entsprechenden Änderungen in der physischen Beschaffenheit dieses Raums oder vielmehr dieser Räume, die nacheinander und nebeneinander, in erheblichem Maße auch gleichzeitig an denselben Stellen im physischen Raum konstituiert wurden und werden. ${ }^{105}$

Wozu aber werden Räume von Menschen konstituiert - hybride Räume, aber auch die vielen gänzlich virtuellen und metaphorischen Räume? Der hauptsächliche Grund dürfte in der »Ordnungsleistung der Verräumlichung «106 liegen. Martina Löw zufolge dienen Räume der "Organisation des Nebeneinanders «; ${ }^{107}$ entsprechend definiert sie: "Raum ist eine relationale (An)Ordnung von Lebewesen und sozialen Gütern «, ${ }^{108}$ wobei auch die Güter primär materiell oder primär symbolisch sein können. ${ }^{109}$ Raum strukturiert also die Verhältnisse von Menschen untereinander wie auch von Menschen zu Gütern, die für sie von Bedeutung sind. Daraus folgt, dass Raumfragen sehr häufig mit Fragen von Macht und Ungleichheit verknüpft sind. ${ }^{110}$ Über die Konstitution von Räumen wird etwa bestimmt, wer mit wem in Kontakt treten kann, wer sich dem Zugriff von anderen entziehen kann, wer Zugang zu Gütern hat und wer von ihnen ferngehalten wird; die Zuschreibung dieser Setzungen an einen Raum dient häufig dazu, sie zu entpersonalisieren und zu reifizieren. ${ }^{111}$ Darüber hinaus ist auch die Möglichkeit, Räume zu bilden, respektive die Chance, sich bei der Bildung von Räumen gegen andere durchzusetzen, alles andere als gleichmäßig verteilt. Henri Lefebvre hat dies in neomarxistischer Begrifflichkeit so formuliert, dass die Produktion von Raum ("production 
de l'espace«) von Produktivkräften und Produktionsverhältnissen ebenso bestimmt werde wie jede Erzeugung von Gütern. ${ }^{112}$

Wichtig zu bemerken ist auch, dass Räume nicht statisch sind. Einmal konstituiert, erhalten sie sich nicht etwa gleichsam von selbst - schon deshalb nicht, weil Menschen und Güter in ständiger Bewegung sind. Deshalb "konstituiert sich [Raum] auch in der Zeit«. ${ }^{113}$ Oft werden die räumlichen Strukturen dabei durch repetitives Handeln in gewohnten Routinen ohne viel Abweichung reproduziert, ${ }^{114}$ es können aber auch Veränderungen eintreten - unmerkliche im Laufe der Zeit und gelegentlich auch einschneidende, wenn bestehende Räume grundlegend verändert oder ganz neue Räume geschaffen werden. Letzteres erfordert besonders hohen Ressourceneinsatz und setzt nicht selten auch beträchtliches Konfliktpotenzial frei. Schließlich können Räume sich auch auflösen oder vorsätzlich zerstört werden. ${ }^{115}$

Räume können in verschiedensten Größenordnungen gedacht werden - von der gesamten von Menschen wahrnehmbaren Welt über Kontinente, Regionen oder Siedlungen bis hinab zum Zimmer, zur Schachtel oder Flasche und nicht zuletzt zum menschlichen Körper, der sich gleichfalls als Raum verstehen lässt. ${ }^{116}$ Dementsprechend können Räume andere Räume in beliebiger Zahl enthalten, was manchmal als hierarchische Überordnung gedeutet wird; sie können sich mit anderen Räumen überschneiden. Der Begriff des `Ortes` wird bei verschiedenen Autorinnen und Autoren oft, aber in uneinheitlicher Weise von jenem des 'Raums den, etwa um innerhalb des Raums als Anordnung die einzelne, benennbare Stelle zu bezeichnen, an der ein Mensch oder Objekt platziert sein kann. ${ }^{117}$

Das Verhältnis des modernen Staats zum Raum wird oft mit dem Begriff der >Territorialität « umschrieben. ${ }^{118}$ Die Anfänge dieses Konzepts lassen sich bis in die frühneuzeitliche Rechtslehre zurückverfolgen. ${ }^{119}$ Gemeint ist, dass als Geltungsbereich der legitimen Machtausübung des Staates ein geographischer Raum festgelegt ist, innerhalb dessen sie möglichst ausschließlich und möglichst einheitlich sein soll. `Ausschließlichkeit` bedeutet, dass nur die Agenten und Agentinnen des Staates jene Machtmittel gebrauchen sollen, auf die für den Staat ein Monopolanspruch erhoben wird. 'Einheitlichkeit` meint, dass die staatlichen Normen in allen Teilen des Territoriums gleiche Geltung haben und die staatliche Machtausübung in der gleichen Weise erfolgen soll. Beides setzt die Eindeutigkeit und Fixität des Territoriums voraus, also lineare, verbindlich und exakt festgeschriebene sowie anerkannte Grenzen. ${ }^{120}$ In historischer Perspektive sind freilich alle diese Merkmale als Ansprüche zu sehen, die in Staatstheorien erhoben und von Trägern und Trägerinnen staatlicher Macht verfolgt, aber nur allmählich und oft nicht vollständig realisiert wurden.

Durchaus im Sinne der Erneuerung des Raumbegriffs haben neuere Forschungen zum Staat betont, dass das Territorium eines Staates als Raum, von dem sich sinnvoll sprechen lässt, erst im Laufe der Staatsbildung konstituiert wird. Dies meint nicht nur die militärische Eroberung oder vertragliche Inbesitznahme von Gebietsteilen, sondern auch und vor allem die tatsächliche Etablierung einer kontinuierlichen und einheitlichen staatlichen Herrschaft durch Verwaltung, die Erschließung durch Kommunikationswege und nicht zuletzt die Erzeugung eines Wissens vom Staatsgebiet als Einheit, sowohl für den internen Gebrauch staatlicher Institutionen als auch zur weiteren Verbreitung. ${ }^{121}$ Mit alldem geht auch die sich verfestigende Neigung zu einem 'geopolitischen ¿ Denken einher, in welchem politische Macht "ausschließlich in räumlichen Kategorien gemessen " wird. Solche Macht »manifestiert sich sowohl in der Herrschaft des Staates über sein eigenes Territorium als auch in seinen Möglichkeiten zu territorialer Expansion, wobei die geographischen Gegebenheiten im eigenen Land Lage, Ausdehnung, Bevölkerungsgröße und -struktur, Ressourcen etc. - die Chancen im globalen Machtkampf festlegen «. ${ }^{122}$ In vielen Fällen ist mit den genannten Entwicklungen auch die Konstruktion des Staatsvolks als Schicksalsgemeinschaft, als >Nation`, parallel und eng verflochten, worauf in diesem Rahmen nicht weiter eingegangen werden kann. ${ }^{123}$

Durch staatliches Wirken konstituiert oder von ihm betroffen werden jedoch ebenso diverse weitere Räume innerhalb und - ungeachtet des Ausschließlichkeitspostulats - auch außerhalb des Territoriums, wie die französischen Konsulate im Osmanischen Reich, die Mathieu Jestin in seinem Beitrag behandelt. Manche Räume werden in besonders augenfälliger Weise für den Staat errichtet und sind ihm dann explizit zugeordnet; dazu zählen Provinzen und Verwaltungsbezirke, jedoch können etwa auch Amtsgebäude und Kasernen, Poststationen, Schulhäuser oder Bahnhöfe bei maßgeblicher Rolle 
des Staates in diesen Bereichen einen ähnlichen Charakter annehmen, ebenso Kulturgutspeicher wie Archive oder Museen sowie Denkmäler und öffentliche Plätze, wenn sie als Stätten staatlicher Repräsentation genutzt werden. Darüber hinaus aber bedingt die wachsende und sich verdichtende Regelungstätigkeit staatlicher Stellen für immer mehr Bereiche des gesellschaftlichen Lebens fast unweigerlich Einflussnahmen auf die Konstitution und Nutzung von Räumen. Wenn etwa die Sicherung von Rechten und Besitz der Einzelnen zunehmend als Zuständigkeit an den Staat gezogen wird, erfordert dies die Lokalisierung materieller Besitztümer in Grundbüchern, Katastern oder Inventaren. Wenn im Namen des Staates die Mobilität von Menschen, Gütern und Information - es handle sich um Reisen, um Migration, um Handel, Briefverkehr oder Telefonanschlüsse - mal gefördert und mal eingeschränkt, jedenfalls reguliert und kontrolliert wird, dann werden dadurch Räume hergestellt, integriert, zerteilt, abgeschottet, wie sich etwa im Artikel von Nadja Weck am Beispiel des Beitrags der Eisenbahn zur Konstitution der habsburgischen Provinz Galizien als Raum zeigt. Im 20. Jahrhundert kommt es zur >Raumordnung` und >Raumplanung`, die verschiedenste Arten von Raumnutzungen auch prospektiv für die Zukunft unter staatliche Aufsicht stellt. ${ }^{124}$ Einem richtungsweisenden Werk der diesbezüglichen Literatur, nämlich der Habilitation von Frido Wagener, widmet Margrit Seckelmann eine Relektüre in diesem Band.

Diese und weitere Vorgänge der Konstitution, Gestaltung und Nutzung von Räumen unter wie auch immer gearteter Beteiligung von dem Staat zugeordneten Akteuren und Aktanten sind Forschungsgegenstände im Sinne des Themas für den vorliegenden Band. Dabei können sich aus der Verknüpfung mit den in den vorangegangenen Abschnitten dargelegten Überlegungen $\mathrm{zu}$ Staat und Verwaltung vielfältige Fragestellungen ergeben. Beispielsweise kann gefragt werden, wie staatliche Zuständigkeit für bestimmte Räume und raumbezogene Regelungsleistungen kommunikativ erzeugt und legitimiert wurde. Es kann den Interaktionen zwischen dem Staat zugeordneten und anderen Akteuren und Akteurinnen bei der Konstitution und Nutzung von Räumen nachgegangen werden in der Annahme, dass auch im 19. Jahrhundert ein ausgehandelter Herrschaftsvollzug weitaus eher die Regel war als eine restlose Durchsetzung staatlicher Anordnungen. Die Konfigurationen von
Interessen und Motiven sind dabei wohl meist vielfältiger zu denken denn als einfache Konfrontation von staatlichem Streben nach Durchgriff mit Resistenz oder notgedrungener Akzeptanz der Betroffenen. Es können die intellektuellen und materiellen Werkzeuge, die bei der Konstitution von Räumen zum Einsatz kamen, auf ihren Ursprung, ihre Eigenschaften und Wirkungen hin untersucht werden. Ausweitungen wie auch Rücknahmen und qualitative Veränderungen der Rolle des Staates können als unintendierte Konsequenzen von Verläufen oder von Technologien ebenso gut wie als Realisierung bewusst angestrebter Ziele - sei es auf Seiten der im Namen des Staates Handelnden oder ihrer Gegenüber gedeutet werden.

Früh und richtungsweisend untersucht hat die Verbindung von Mediengebrauch, Raum und Staatlichkeit respektive Verwaltung der kanadische Wirtschaftswissenschaftler und von Marshall McLuhan als Lehrer verehrte Harold A. Innis. In seinem 1951 erschienenen Sammelwerk "The Bias of Communication « widmete er einen Aufsatz dem »Problem des Raumes«. In einem aphoristischen Husarenritt durch mehr als 6000 Jahre Geschichte gibt Innis zu verstehen, dass Staatsgebilde je nach Medieneinsatz entweder $\mathrm{zu}$ »zentralisierenden Formen " tendieren, "die die Herrschaft im wesentlichen statisch im Raum artikulieren und (mühsam) gegen die Zeit stabilisieren«, oder zu »dezentralisierenden Formen, die sich eher dynamisch in der Zeit artikulieren und (mühsam) im Raum halten «. ${ }^{125}$ Im Hinblick auf die europäische Neuzeit deklamiert Innis: „Papier- und Druckindustrie förderten die Entwicklung von räumlichen Monopolen in Form von Nationalismus und Staat «. ${ }^{126}$ Wolfgang Hagen hat das methodische Prinzip von Innis' Geschichtsdeutung in der Suche nach medienbasierten Raum- und Zeitmonopolen gesehen, weil Innis den Gang der Geschichte konkurrenzlos aus der Abfolge neuer Medien und ihren Wirkungen ableitete: „Die massive Ausbreitung der Presse in der zweiten Hälfte des 19. Jahrhunderts, das Anwachsen des ersten sschnellen` (weil täglichen und mehrmals täglichen) Massenmediums Zeitung in den zwanziger Jahren hat zu einer Uniformisierung der Kultur und zu einem territorialen Expansionismus des Staats beigetragen «. ${ }^{127}$ Nationalstaaten, diese Assemblage aus uniformierter Kultur und territorial expandierendem Staat, sind in einer solchen Lesart reine Effekte der Medientechnologien. 
Gewissermaßen in Anlehnung an das methodische Prinzip von Innis und in Fortführung und Erweiterung desselben untersuchen die vorliegenden Beiträge nicht nur Medienmonopole, sondern multiple slittle tools` wie auch slarger tools` der Verwaltung auf ihre raumordnenden Effekte. Im Falle administrativer Schriftlichkeit beispielsweise ist es nicht ihr bloßer Einsatz allein, der Räume konstituiert, sondern vielmehr ihre spezifische Gebrauchsweise, wie Birgit Näther in ihrem Beitrag ausführt. Indem bayerische Mittelbehörden praktisch vorgaben, wie Visitationen vorzunehmen waren, verstrickten sie die ihnen übergeordneten Zentralbehörden nicht nur in eine gemeinsame Verfahrensgeschichte, sondern erschlossen auch einen gemeinsamen, nicht nur papierenen Verwaltungsraum. Indem die Oberbehörden die Vorlagen der Mittelbehörden übernahmen, definierten sie die Praxis der Mittelbehörden schließlich als Verfahrensnorm.

In Bezug auf Verwaltung kann weiter gefragt werden, ob administratives Handeln bestimmte Arten von Räumen erfordert respektive gewisse Räume ein bestimmtes administratives Handeln evozieren. So zeigt Josef Löffler in seinem Beitrag, wie sehr im habsburgischen Vormärz eine vom Staat angeordnete, aber von Patrimonialherrschaften durchgeführte Verwaltung an den Umstand angepasst war, dass ihre Bezugsräume am gewachsenen Besitzstand der Herrschaften und nicht am staatlichen Bedürfnis nach Vereinheitlichung und Übersichtlichkeit des Territoriums orientiert waren. Thomas Stockinger analysiert in seinem Beitrag, an Cornelia Vismann anschließend, wie die namensgebende Schranke in den Kanzleien ein Interieur einrichtete, in dem Kontakte zwischen Beamten und Verwalteten, zwischen dem Inneren der staatlichen Sphäre und ihrer Umwelt, "nicht unterbunden, sondern vielmehr lokalisiert, reglementiert und hierarchisiert « wurden. Auch die sprichwörtlichen Korridore der Verwaltung waren weit davon entfernt, einfach nur Schikanen zu sein, sie steuerten den Verkehr von Bittstellern ohne Störung der arbeitenden Beamten.

Nicht nur haben Räume Wirkungen auf die Verwaltung. Umgekehrt haben administrative Praktiken auch unmittelbare oder mittelbare räumliche Effekte, etwa wenn bürgerliche Beamte herzogliche Territorien besuchten, um Verfahren in Gang zu bringen, wie Birgit Näther schildert. Josef Löffler weist darauf hin, wie Stra- das heißt als Komponenten bestimmter Räume markiert wurden, welche damit ihrerseits Sichtbarkeit erlangten. Thomas Stockinger stellt fest, dass Orte aufgrund ihrer zeitlichen - statt geographischen - Nähe zum Amtssitz bestimmten Bezirken zugeschlagen wurden; wie Beamte Räume verkleinerten, indem sie Orte bereisten und Bürgern die Verwaltung wortwörtlich näher brachten, führt Rüdiger von Krosigk in seinem Beitrag aus. Administrative Praktiken zeitigten dabei nicht nur räumliche, sondern auch staatliche Konsequenzen. Staatlich wurden jene Akte allerdings nicht schon deshalb, weil sie administrativ waren, sondern weil sie im Auftrag einer zentralen Staatsgewalt ergingen, das heißt delegiert wurden. Einander ergänzend zeigen Josef Löffler und Thomas Stockinger in ihren Texten, wie die habsburgische Staatsspitze den Grundherrschaften ihre administrativen Kompetenzen entzog, um sie von ihr selbst errichteten Bezirksverwaltungen zu übertragen, was wiederum dazu führte, dass staatliche Verwaltung lokal wurde.

Zur Strukturierung der Vielfalt von Vorgängen, in denen Räume konstituiert wurden, möchten wir eine Dreiheit von Aspekten anbieten: Raumbildung durch Grenzen, Raumbildung durch Wissen und Raumbildung durch Nutzung. Wir sind uns dessen bewusst, dass es sich dabei um alles andere als eine trennscharfe Unterscheidung handelt. Die drei raumbildenden Faktoren treten in aller Regel gemeinsam auf, gehen ineinander über, ja setzen einander nicht selten gegenseitig voraus. In den meisten Beiträgen dieses Bandes kommen auch mehrere von ihnen zur Sprache. Dennoch steht in einzelnen Texten der ein oder andere dieser Aspekte erkennbar im Mittelpunkt, was teils an den besprochenen Gegenständen, teils an den gewählten Zugängen liegt.

Das Ziehen von Grenzen ist der vielleicht augenfälligste Akt der Herstellung von Räumen und wird in mehreren unserer Artikel in den Mittelpunkt gestellt. Die Grenze ist als Konzept wie in ihrer lebensweltlichen Realisierung stets von einer fundamentalen Ambivalenz geprägt: Sie »verbürgt [...] zwar die Identität mindestens zweier Bereiche, führt das Getrennte jedoch zugleich einander zu und lässt es in wechselseitiger Abhängigkeit erscheinen «. ${ }^{128}$ Der Bildung und Erhaltung von Identität dient sie, indem sie das von ihr Umschlossene (ungeachtet seiner möglichen Heterogenität) als Einheit zu definieren erlaubt und aus der Perspektive derjenigen, die selbst innerhalb der Grenze verortet sind, als 'Eigenes، 
bestimmt. Dies ist natürlich nur denkbar in Unterscheidung vom `Anderen` oder `Fremden`, das jenseits der Grenze liegen soll. ${ }^{129}$ Gleichzeitig sind aber die wenigsten Grenzen zwischen von Menschen konstituierten Räumen ganz undurchdringlich; vielmehr bedingen sie jeweils bestimmte Modalitäten des erlaubten und kontrollierten sowie des verbotenen, aber doch stattfindenden Grenzübertritts. Sie sind mithin Instrumente der "Regulierung von Mobilität«, ${ }^{130}$ nicht der Unterbindung derselben. Daher ist die Untersuchung der »Voraussetzungen, Bedingungen und Folgen « von Grenzüberschreitungen ein vielversprechender Weg zur Erforschung von Bedeutung und Zweck der Grenze selbst. ${ }^{131}$ Interessant ist auch, wie Grenzen materiell und symbolisch manifestiert wurden: durch Marken im Gelände, durch Sperren an Übergängen, durch Grenzbeschreibungen und Karten. Gerade wenn ihre materielle Wahrnehmbarkeit gering war, was eher die Regel als die Ausnahme darstellt, bedurften sie der Verankerung im Bewusstsein und der Anerkennung derer, die sich nach ihnen richten sollten. Vor allem bei neu gezogenen oder verschobenen Grenzen konnte dies beträchtlicher Anstrengungen sowie einer langen Zeit bedürfen. ${ }^{132}$ Den Betroffenen wurde eine Änderung ihres Raumwissens und ihrer Raumnutzung abverlangt, zu der viele nicht ohne Weiteres bereit waren, wie Ellinor Forster in ihrem Aufsatz beschreibt.

Für das Anliegen unseres Bandes kommen Grenzen ganz unterschiedlicher Art in Betracht, bei den Staatsgrenzen angefangen über Grenzen von Verwaltungseinheiten bis zur Türschwelle der Amtsstube oder zu der - von Thomas Stockinger in seinem Beitrag diskutierten - Schranke, die darin den Bereich der Beamten von dem schied, den die Verwalteten betreten durften. Von Interesse ist dabei einerseits das Ziehen neuer Grenzen, ein Vorgang, der auf vielfältigen Ebenen verlaufen kann - Debatten in Parlamenten und Fachzeitschriften, wie sie Luigi Blanco in seinem Artikel für Italien analysiert, gehören ebenso dazu wie das Abbauen und Versetzen eines Zauns, das in Ellinor Forsters Aufsatz eine Rolle spielt. Andererseits kann auch der Umgang mit bestehenden Grenzen ins Auge gefasst werden, ihre Erhaltung, Überwachung, Beschreibung sowie ihre erlaubte oder unerlaubte Überschreitung. Dass räumliche Ordnungen und Netzwerke auch über staatliche Grenzen hinweg gebildet werden und funktionieren konnten, ohne sich doch dem Zugriff staatlicher Verwaltung ganz zu entziehen, beweist der Beitrag von Nathalie Patricia Soursos und Anna Ransmayr über griechisch-orthodoxe Stiftungen in Wien, die im Osmanischen Reich und anderen Balkanstaaten ihre Tätigkeit entfalteten. Nicht zuletzt erscheinen auch die in den vorangegangenen Abschnitten angesprochenen Grenzen zwischen Staat und nichtstaatlicher Sphäre, zwischen Verwaltung, anderen Staatstätigkeiten und Zivilgesellschaft als lohnende Untersuchungsgegenstände - nicht im Hinblick auf eine objektive oder normative Bestimmung, wo sie liegen, sondern auf die Historisierung ihrer Setzung.

Einen zweiten Zugriff auf die Raumbildung bietet das Raumwissen. Darunter ist alles Wissen zu verstehen, das Räume und das in ihnen Enthaltene beschreibt, erfasst und somit auffindbar und administrativem Handeln zugänglich macht. Dieses Wissen bildet aber nicht etwa nur vorgängig existente Räume $a b$, sondern es erschafft sie selbst, indem es ihnen Quantitäten und Qualitäten zuschreibt, Namen oder Adressen gibt und sie zu Bezugsgrößen des Denkens, Sprechens und Handelns macht, ${ }^{133}$ wie dies etwa Rüdiger von Krosigk für die badischen Bezirke oder Stefan Couperus, Harm Kaal, Nico Randeraad und Paul van Trigt für die Provinz Südholland zeigen. Nicht selten sind auch räumliche Ordnungen gerade mit der Absicht erst hergestellt worden, als Raster für die Erhebung und Zuordnung von Wissen über Menschen und Güter zu dienen, wie etwa Anton Tantner für die Häusernummerierung gezeigt hat. ${ }^{134} \mathrm{Zu}-$ gleich mit der möglichst präzisen und eindeutigen Lokalisierung und Adressierung der erfassten Menschen und Güter tendierte die Erhebung von Wissen für den Staat und seine Verwaltung zur Standardisierung und Kategorisierung. Dies hing mit der Zweckorientierung der Wissensproduktion zusammen, die nicht alle Menschen und Güter verwertbar und somit erhebenswert fand, sondern nur manche, und auch an diesen nur bestimmte Eigenschaften; ${ }^{135}$ es lag aber auch an dem spezifischen Verhältnis des Staats zu seinem Raum, nämlich an dem Ziel einer einheitlichen Herrschaftsausübung. Hieraus ergab sich jener besondere Modus der Wahrnehmung, für den James C. Scott den Ausdruck »seeing like a state " geprägt hat. ${ }^{136}$

Viele Teilbereiche des Staatswissens und der Staatswissenschaften, welche seit dem ausgehenden 18. Jahrhundert in rasanter Weiterentwicklung standen, hatten deutliche Raumbezüge. Dazu zählten neben dem bereits 
erwähnten Volkszählungswesen und der amtlichen Statistik insgesamt, die sich von der verbal-deskriptiven zur quantitativen Wissenschaft von den Eigenschaften und Ressourcen des Staates entwickelte, ${ }^{137}$ der amtlichen Bodendokumentation in Katastern und Grundbüchern $^{138}$ sowie der Kartographie ${ }^{139}$ im Grunde sämtliche Formen des administrativen Berichtswesens, insofern sie sich an den Verwaltungseinheiten als Bezugsgrößen orientierten. Die Erfordernisse des regelmäßig eingeforderten Berichtens trugen ihrerseits viel dazu bei, den Ausbau von Verwaltungskapazität und die Verfestigung von Praxis und Routinen auf der lokalen Ebene voranzutreiben, ${ }^{140}$ wie dies etwa in den Texten von Birgit Näther, Anette Schlimm, Josef Löffler und Rüdiger von Krosigk zum Ausdruck kommt. Die raumbezogen organisierte Verwaltung erzeugte unweigerlich Raumwissen; wie sie dies tat, aus welchen Quellen sie es bezog, welche Formen sie ihm gab und wie es wiederum in ihr Handeln einfloss, lohnt allerdings einer näheren Untersuchung.

Als dritten Bereich der Raumkonstitution - wiederum mit unscharfer Abgrenzung gegen die beiden anderen - möchten wir die Nutzung von Räumen respektive das Handeln in Räumen vorstellen. Hier stehen nicht explizite Akte der Raumbildung durch Grenzziehung oder durch Beschreibung im Fokus, sondern jene Reproduktion, Erhaltung oder allmähliche Umformung von Räumen, die sich aus jedem auf sie bezogenen menschlichen Handeln ergibt. In diesem Zusammenhang aufschlussreich ist etwa der Befund, dass in Bürokratien mehr und mehr "am Schreibtisch im Amtszimmer, und nicht - wie beim Kollegialprinzip die Regel - am Beratungstisch im Konferenzzimmer verwaltet« wird. ${ }^{141}$ Hier ist die raumbildende Wirkung den beteiligten Akteuren meistens nicht bewusst, und allfällige Veränderungen treten oft so langsam ein, dass sie erst im historischen Rückblick sichtbar werden. Dennoch ist dieser Aspekt stets präsent, weil nicht nur - wie eingangs festgehalten - der Staat, seine Institutionen und Tätigkeiten einen unhintergehbaren Raumbezug haben, sondern grundsätzlich alles menschliche Handeln in Räumen stattfindet, von diesen beeinflusst wird und auf sie wirkt. Somit geht es bei diesem dritten Bereich nicht darum, Teilbereiche des Verwaltungshandelns aufzusuchen, die besonders klare Raumbezüge haben, vielmehr kann jeder Aspekt der Verwaltungsgeschichte auf solche Bezüge hin gelesen werden. In pragmatischer Hinsicht mag diese dritte
Unterteilung als Restkategorie erscheinen, wir halten sie aber insofern für sinnvoll, als das Verhältnis von Staat und Verwaltung zu Raum eben nicht vollständig in jenen Situationen aufgehoben ist, in denen zielgerichtet auf die Bildung und Beschreibung von Räumen hingewirkt wird.

\section{Schlüsse}

Es mag bei der Lektüre des Vorangegangenen aufgefallen sein, dass unsere Ausführungen zu Staat, Verwaltung und Raum eine Reihe von Parallelen aufweisen, die sich zum erheblichen Teil aus der Theorieentwicklung und Forschungsgeschichte notwendig ergeben, in gewissem Maße aber selbstverständlich auch unserer eigenen Positionierung und Perspektive entspringen. So haben wir bei jedem der drei untersuchten Begriffe auf Reifizierungen hingewiesen, welche die ältere Theoriebildung dominierten und den alltagssprachlichen Gebrauch aller drei Termini weiterhin prägen, von denen wir uns aber wie auch der überwiegende Teil der aktuellen einschlägigen Forschung distanzieren wollen. Wir haben gezeigt, dass zu diesen Reifizierungen zwangsläufig die Vorstellung von eindeutigen und `natürlichen`Grenzen gehört, die sich aber bei näherer Reflexion als intentional gesetzt und oft als sachlich fragwürdig erweisen. Wir haben demgegenüber Staat, Verwaltung und Raum statt als in sich geschlossene Phänomene einerseits hinsichtlich ihres Zustandekommens als soziale und kulturelle Konstrukte, andererseits hinsichtlich ihrer Beschaffenheit als relationale Anordnungen oder Assemblagen von Menschen und Dingen beschrieben, die durch Bedeutungszuweisungen und Praktiken zusammengehalten werden. Dabei sind Staat und Verwaltung durch die Arten der angeordneten Elemente und ihrer Verbindungen, vor allem aber durch bestimmte Zwecke der Anordnung näher spezifiziert; sie erscheinen somit als Sonderfälle von Raum. Anders gesagt: Aus unseren Überlegungen ergibt sich, dass Staat und Verwaltung nicht nur in Räumen existieren, Räume konstituieren und nutzen, sondern dass beide selbst Räume sind.

Im Umkehrschluss folgt aus dieser Feststellung, dass Staat und Verwaltung ohne Räume nicht zu haben sind. Räume sind nicht einfach Akzidenzien von Staat und Verwaltung, sondern unverzichtbare Teile dieser Assemblagen. Arrondierung und Territorialisierung stellen 
wesentliche Entstehungsprozesse moderner Staaten dar, deren Bürokratisierung zu wesentlichen Teilen ihre namengebenden Räume, die Büros, ermöglichen. Wenn sich Räume verändern, ändern sich infolgedessen auch Staaten und Verwaltungen. Das rückt die Frage in den Mittelpunkt, wie das Verhältnis von Staaten und Räumen, Verwaltungen und Räumen weiter zu denken ist. Auf welche Art und Weise verändern sich Staaten und Verwaltungen, wenn sich deren Räume verändern? Und wie verändern sich umgekehrt die Räume von Staaten und Verwaltungen, wenn sich Staaten oder Verwaltungen verändern?

Solche Befunde und Fragen tauchen am Ende dieser Einleitung als jene Gedanken auf, welche die zutiefst unterschiedlichen Forschungsgegenstände und Ansätze der einzelnen Beiträge verbinden und somit, über das gemeinsame Freischaltungsdatum hinaus, den zweiten Jahrgang von "Administory« als Band zusammenhalten. Kein einzelner Text, auch nicht der Band in seiner Gänze, vermag sämtliche denkbaren Konsequenzen und Verästelungen dieser Gedanken im Detail zu verfolgen. Doch bleibt es unsere Hoffnung, dass die Vielfalt dessen, was die Autorinnen und Autoren zu Staat, Verwaltung und Raum sowie den Verbindungen zwischen ihnen vorbringen, auf die eben skizzierten Zusammenhänge rückführbar ist und diese Sammlung von Texten somit einen nicht nur reichhaltigen, sondern auch kohärenten Beitrag zur Vertiefung des Verständnisses für diese leisten wird. 


\section{Publikationen mit Quellencharakter}

B E R G , Günther Heinrich von: Handbuch des Teutschen Policeyrechts, Teil 1, Hannover ${ }^{2} 1802$. J u s T I , Johann Heinrich Gottlob von: Grundsätze der Policeywissenschaft in einem vernünftigen, auf den Endzweck der Policey gegründeten, Zusammenhange zum Gebrauch Academischer Vorlesungen abgefasset, Göttingen ${ }^{3} 1782$. M A Y ER, Otto: Deutsches Verwaltungsrecht, Bd. 1, Leipzig 1895. MEYER, Georg: Lehrbuch des deutschen Staatsrechtes, Leipzig 1878. M o N T E S Q U I E U, Charles-Louis de Secondat de: De I'Esprit des lois, Bd. 1, London 1777. W I L s o N, Woodrow: »The Study of Administration «, in: Political Science Quarterly 2 (1887), S. 198-222. Z E D L E R, Johann Heinrich (Hg.): Grosses vollständiges Universal-Lexicon aller Wissenschaften und Künste, Bd. 30, Leipzig 1741.

\section{Sekundärliteratur}

А в в от т, Kenneth W. / S N I AL, Duncan: »Hard and Soft Law in International Governance«, in: Judith L. Goldstein et al. (Hg.): Legalization and World Politics, Cambridge (Massachusetts) 2001, S. 37-72. A g A R, Jonathan: The Government Machine. A Revolutionary History of the Computer, Cambridge (Massachusetts) 2003. A L L I è S, Paul: L'invention du territoire, Grenoble 1980. A N T E R, Andreas: Max Webers Theorie des modernen Staates. Herkunft, Struktur und Bedeutung, Berlin 1995. B A E C K E R, Dirk: »Durch diesen schönen Fehler mit sich selbst bekannt gemacht«, in: Dirk Baecker: Organisation und Management. Aufsätze, Frankfurt am Main 2003, S. 18-40. B A L K E, Friedrich / S I E GERT, Bernhard / V O G L, Joseph (Hg.): »Medien der Bürokratie«, in: Archiv für Mediengeschichte 16 (2016). B A R T E L S O N , Jens: The Critique of the State, Cambridge 2001. BAYLY, Christopher A.: The Birth of the Modern World 1780-1914. Global Connections and Comparisons, Malden 2004. B E H R I S CH , Lars: "Vermessen, Zählen, Berechnen des Raums im 18. Jahrhundert «, in: Lars Behrisch (Hg.): Vermessen, Zählen, Berechnen. Die politische Ordnung des Raums im 18. Jahrhundert, Frankfurt am Main 2006, S. 7-25. B E N T L EY, Michael: »Boundariess in Theoretical Language about the British State«, in: Simon J. D. Green / Richard C. Whiting (Hg.): The Boundaries of the State in Modern Britain, Cambridge 1996, S. 2956. B E N Z, Arthur: Der moderne Staat. Grundlagen der politologischen Analyse, München 2001. • / D o S E, Nicolai (Hg.): Governance - Regieren in komplexen Regelsystemen. Eine Einführung, Wiesbaden ${ }^{2} 2010$. B L A I R, Ann M.: Too Much to Know. Managing Scholarly Information before the Modern Age, New Haven 2010. B o G D A N D Y, Armin von / D A N , Philipp: »International Composite Administration: Conceptualizing MultiLevel and Network Aspects in the Exercise of International Public Authority", in: German Law Journal. Developments in German, European and International Jurisprudence 9 (2008), S. 2013-2039. B o U R D I E U , Pierre: Sur l'État. Cours au Collège de France (1989-1992), hg. von Patrick Champagne et al., Paris 2012. B RA D D I C K, Michael J.: State Formation in Early Modern England c. 1550-1700, Cambridge 2000. • "State Formation and Political Culture in Elizabethan and Stuart England. Micro-histories and Macro-historical Change«, in: Ronald G. Asch / Dagmar Freist (Hg.): Staatsbildung als kultureller Prozess. Strukturwandel und Legitimation von Herrschaft in der Frühen Neuzeit, Köln 2005, S. 69-90. B R E D E K A M P, Horst: Thomas Hobbes visuelle Strategien. Der Leviathan: Urbild des modernen Staates. Werkillustrationen und Portraits, Berlin 1999. B RE N N A N, Catherine: Max Weber on Power and Social Stratification. An Interpretation and Critique, Aldershot 1997. B R E U E R, Stefan: Der Staat. Entstehung, Typen, Organisationsstadien, Reinbek 1998. B R Ü C K W E H , Kerstin: Menschen zählen. Wissensproduktion durch britische Volkszählungen und Umfragen vom 19. Jahrhundert bis ins digitale
Zeitalter, Berlin 2015. B R U N N E R, Walter: „Historische Bodendokumentation und die Anfänge der Katastralvermessung in Mitteleuropa. Ein Überblick«, in: Mitteilungen des Steiermärkischen Landesarchivs 47 (1997), S. 89-105. C A N C I K, Pascale: „Verwaltung, Raum, Verwaltungsraum - eine historische Annäherung", in: Hermann Hill / Utz Schliesky (Hg.): Die Vermessung des virtuellen Raums. E-Volution des Rechts- und Verwaltungssystems III, Baden-Baden 2012, S. 29-54. C h E V A L I E R, Jacques: Science administrative, Paris ${ }^{3} 2002$. C H R I S T M A N N, Gabriela B.: »Das theoretische Konzept der kommunikativen Raum(re)konstruktion«, in: Gabriela B. Christmann (Hg.): Zur kommunikativen Konstruktion von Räumen. Theoretische Konzepte und empirische Analysen, Wiesbaden 2016, S. 89-117. C L A N C H Y, Michael: "Does Writing Construct the State?«, in: The Journal of Historical Sociology 15 (2002), S. 68-70. C L A V A L, Paul: Les espaces de la politique, Paris 2010. D A M K o w S K I, Wulf: Die Entstehung des Verwaltungsbegriffes. Eine Wortstudie, Köln 1969. D A N N E B E R G , Lutz: Die Anatomie des Text-Körpers und NaturKörpers. Das Lesen im liber naturalis und supernaturalis, Berlin 2003. D E L A N D A, Manuel: A New Philosophy of Society. Assemblage Theory and Social Complexity, London 2006. DE S R o S İe RES, Alain: La politique des grands nombres. Histoire de la raison statistique, Paris 1993. D I N G E S, Martin: »Aushandeln von Armut in der Frühen Neuzeit: Selbsthilfepotential, Bürgervorstellungen und Verwaltungslogiken«, in: WerkstattGeschichte 10 (1995), S. 7-15. • "Justiznutzungen als soziale Kontrolle in der Frühen Neuzeit«, in: Andreas Blauert / Gerd Schwerhoff (Hg.): Kriminalitätsgeschichte. Beiträge zur Sozial- und Kulturgeschichte der Vormoderne, Konstanz 2000, S. 503-544. D ö R I N G , Jörg / T H I E L M A N N, Tristan: „Einleitung: Was lesen wir im Raume? Der Spatial Turn und das geheime Wissen der Geographen«, in: Jörg Döring / Tristan Thielmann (Hg.): Spatial Turn. Das Raumparadigma in den Kultur- und Sozialwissenschaften, Bielefeld 2008, S. 7-45. E D N E Y, Matthew H.: Mapping an Empire. The Geographical Construction of British India, 1765-1843, Chicago 1997. E I в A C H , Joachim: „Verfassungsgeschichte als Verwaltungsgeschichte«, in: Joachim Eibach / Günther Lottes (Hg.): Kompass der Geschichtswissenschaft, Göttingen 2002, S. 142-151. E L L W E I N , Thomas: Der Staat als Zufall und als Notwendigkeit. Die jüngere Verwaltungsentwicklung in Deutschland am Beispiel Ostwestfalen-Lippe, 2 Bde., Opladen 19931997. E N G E L L, Lorenz: „Wege, Kanäle, Übertragungen. Zur Einführung«, in: Claus Pias et al. (Hg.): Kursbuch Medienkultur. Die maßgeblichen Theorien von Brecht bis Baudrillard, Stuttgart 1999, S. 126-133. • / S I E G E R T, Bernhard: »Editorial«, in: Zeitschrift für Medien- und Kulturforschung 1 (2010), S. 5-9. E s M E R, Tolga U.: »Economies of Violence, Banditry and Governance in the Ottoman Empire around 1800«, in: Past and Present. A Journal of Historical Studies 224 (2014), S. 163-199. F o u C A U L T, Michel: "Das Leben der infamen Menschen«, in: Michel Foucault: Schriften in vier Bänden. Dits et Ecrits, Bd. 3, hg. von Daniel Defert / François Ewald / Jacques Lagrange, Frankfurt am Main 2003, S. 309-332. • Geschichte der Gouvernementalität, Bd. 1: Sicherheit, Territorium, Bevölkerung. Vorlesung am Collège de France 1977-78, hg. von Michel Senellart, Frankfurt am Main 2004. F R A N z I U S, Claudio: "Der >Gewährleistungsstaatı - ein neues Leitbild für den sich wandelnden Staat?«, in: Der Staat. Zeitschrift für Staatslehre und Verfassungsgeschichte, deutsches und europäisches öffentliches Recht 42 (2003), S. 493-517. F R E I S T, Dagmar: „Einleitung: Staatsbildung, lokale Herrschaftsprozesse und kultureller Wandel in der Frühen Neuzeit«, in: Ronald G. Asch / Dagmar Freist (Hg.): Staatsbildung als kultureller Prozess. Strukturwandel und Legitimation von Herrschaft in der Frühen Neuzeit, Köln 2005, S. 1-47. F R E V E R T , Ute: »Neue Politikgeschichte«, in: Joachim Eibach / Günther Lottes (Hg.): Kompass der Geschichtswissenschaft. Ein Handbuch, Göttingen 2002, S. 152-164. F R I E D R I C H , Markus: „Das Buch als Theater. Überlegungen zu Signifikanz und Dimensionen der Theatrum-Metapher als frühneuzeitlichem Buchtitel«, in: Theodor Stammen et al. (Hg.): Wissenssicherung, Wissensordnung 
und Wissensverarbeitung. Das europäische Modell der Enzyklopädien, Berlin 2004, S. 205-232. F u s c o, Sandro-Angelo et al.: "Verwaltung, Amt, Beamter«, in: Otto Brunner / Werner Conze / Reinhart Koselleck (Hg.): Geschichtliche Grundbegriffe. Historisches Lexikon der politischsozialen Sprache in Deutschland, Bd. 7, Stuttgart 1992, S. 1-96. G A N Z E N M Ü LLER， Jörg / T ÖNSMEYER， Tatjana: „Einleitung: Vom Vorrücken des Staates in die Fläche. Ein europäisches Phänomen des langen 19. Jahrhunderts«, in: Jörg Ganzenmüller / Tatjana Tönsmeyer (Hg.): Vom Vorrücken des Staates in die Fläche. Ein europäisches Phänomen des langen 19. Jahrhunderts, Köln 2016, S. 7-31. G A R D E Y, Delphine: Écrire, calculer, classer. Comment une révolution de papier a transformé les sociétés contemporaines (1800-1940), Paris 2008. G E P P E R T, Alexander C. T. / J E N SEN, Uffa / W E I N H O LD, Jörn: „Verräumlichung. Kommunikative Praktiken in historischer Perspektive, 1840-1930«, in: Alexander C. T. Geppert / Uffa Jensen / Jörn Weinhold (Hg.): Ortsgespräche. Raum und Kommunikation im 19. und 20. Jahrhundert, Bielefeld 2005, S. 1549. G ö D E R LE , Wolfgang: Zensus und Ethnizität. Zur Herstellung von Wissen über soziale Wirklichkeiten im Habsburgerreich zwischen 1848 und 1910, Göttingen 2016. G R Ü N E, Niels: "Local Demand for Order and Government Intervention: Social Group Conflicts as Statebuilding Factors in Villages of the Rhine Palatinate, c. 1760-1810«, in: Willem Pieter Blockmans et al. (Hg.): Empowering Interactions. Political Cultures and the Emergence of the State in Europe 1300-1900, Farnham 2009, S. 173186. G U G E R L I, David / S P E I C H , Daniel: Topografien der Nation. Politik, kartografische Ordnung und Landschaft im 19. Jahrhundert, Zürich 2002. G Ü N Z E L, Stephan: »Raum - Topographie - Topologie«, in: Stephan Günzel (Hg.): Topologie. Zur Raumbeschreibung in den Kultur- und Medienwissenschaften, Bielefeld 2007, S. 13-29. • "Spatial Turn - Topographical Turn - Topological Turn. Über die Unterschiede zwischen Raumparadigmen«, in: Jörg Döring / Tristan Thielmann (Hg.): Spatial Turn. Das Raumparadigma in den Kultur- und Sozialwissenschaften, Bielefeld 2008, S. 219-237. H A G E N , Wolfgang: „Globale Gegenwartsversessenheit. Annäherungen an Harold A. Innis und seine Theorie einer MedienRaumzeit«, in: Rudolf Maresch / Niels Werber (Hg.): Raum - Wissen Macht, Frankfurt am Main 2002, S. 193-213. H A R T M A N N, Elke: Die Reichweite des Staates. Wehrpflicht und moderne Staatlichkeit im Osmanischen Reich 1869-1910, Paderborn 2016. H E A D Y, Ferrel: Public Administration: A Comparative Perspective, New York ${ }^{5} 1996$. H ö F E R T, Almut: "States, Cities, and Citizens in the Later Middle Ages», in: Quentin Skinner / Bo Stråth (Hg.): States and Citizens. History, Theory, Prospects, Cambridge 2003, S. 63-75. H o L E N S T E I N , André: »Kommunikatives Handeln im Umgang mit Policeyordnungen. Die Markgrafschaft Baden im 18. Jahrhundert«, in: Ronald G. Asch / Dagmar Freist (Hg.): Staatsbildung als kultureller Prozess. Strukturwandel und Legitimation von Herrschaft in der Frühen Neuzeit, Köln 2005, S. 191-208.• »Introduction: Empowering Interactions: Looking at Statebuilding from Below«, in: Willem Pieter Blockmans et al. (Hg.): Empowering Interactions. Political Cultures and the Emergence of the State in Europe 1300-1900, Farnham 2009, S. 1-31. H o L T Z, Sabine: „Wissen und Macht. Territorialstaat und Bildungspolitik im 17. Jahrhundert«, in: Historisches Jahrbuch 123 (2003), S. 179-196. H U L L, Matthew S.: Government of Paper. The Materiality of Bureaucracy in Urban Pakistan, Berkeley 2012. I N N I S, Harold A.: Empire and Communications, Oxford 1950. • "Das Problem des Raumes« [1951], in: Harold A. Innis: Kreuzwege der Kommunikation. Ausgewählte Texte, hg. von Karlheinz Barck, Wien 1997, S. 147-181. J o y C E, Patrick: The State of Freedom: A Social History of the British State since 1800, Cambridge 2013. K A F K A , Benjamin: "Paperwork: The State of the Discipline«, in: Book History 12 (2009), S. 340-353. • The Demon of Writing. Powers and Failures of Paperwork, New York 2012. K A I N, Roger J. P. / B A I G E N T, Elizabeth: The Cadastral Map in the Service of the State. A History of Property Mapping, Chicago 1992. KA J T Z KE, Laura / S CHROER,
Markus: "Space Studies», in: Stephan Moebius (Hg.): Kultur. Von den Cultural Studies bis zu den Visual Studies. Eine Einführung, Bielefeld 2012, S. 196-215. K E C K, Margaret E. / S I K K I N K, Kathryn: Activists Beyond Borders. Advocacy Networks in International Politics, Ithaca 1998. K E L L E R, Hagen: „Vom sheiligen Buch zur >Buchführung`. Lebensfunktionen der Schrift im Mittelalter«, in: Frühmittelalterliche Studien. Jahrbuch des Instituts für Frühmittelalterforschung der Universität Münster 26 (1992), S. 1-31. K н A R к н о R D I N, Oleg: „What Is the State? The Russian Concept of Gosudarstvo in the European Context«, in: History and Theory. Studies in the Philosophy of History 40 (2001), S. 206-240. K I R S C H, Thomas G.: Spirits and Letters. Reading, Writing and Charisma in African Christianity, New York 2008. K L E I N S C H M I D T, Christoph: „Einleitung: Formen und Funktionen von Grenzen«, in: Christoph Kleinschmidt / Christine Hewel (Hg.): Topographien der Grenze. Verortungen einer kulturellen, politischen und ästhetischen Kategorie, Würzburg 2011, S. 9-21. K o с K A, Jürgen: Unternehmensverwaltung und Angestelltenschaft am Beispiel Siemens 1847-1914. Zum Verhältnis von Kapitalismus und Bürokratie in der deutschen Industrialisierung, Stuttgart 1969. Ko S E L LE C K, Reinhart: Preußen zwischen Reform und Revolution. Allgemeines Landrecht, Verwaltung und soziale Bewegung von 1791 bis 1848, Stuttgart 1967. K RE C K E L, Reinhard: Politische Soziologie der sozialen Ungleichheit, Frankfurt am Main 1992. KR E T z S C H M A R, Dirk: „Region oder Imperium? Zur Semantik von Geopolitik, Raum und Kultur in Russland «, in: Rudolf Maresch / Niels Werber (Hg.): Raum - Wissen Macht, Frankfurt am Main 2002, S. 263-286. L A C R o I X , Bernard: „Ordre politique et ordre social. Objectivisme, objectivation et analyse politique«, in: Madeleine Grawitz / Jean Leca (Hg.): Traité de science politique, Bd. 1: La science politique, science sociale. L'ordre politique, Paris 1985, S. 469565. • „Genèses et constructions de l'État moderne«, in: Antonin Cohen / Bernard Lacroix / Philippe Riutort (Hg.): Nouveau manuel de science politique, Paris 2009, S. 52-70. L A N z I N G E R, Margareth: "Das Lokale neu positionieren im actor-network-Raum - globalgeschichtliche Herausforderungen und illyrische Steuerpolitiken«, in: Ewald Hiebl / Ernst Langthaler (Hg.): Im Kleinen das Große suchen. Mikrogeschichte in Theorie und Praxis. Hanns Haas zum 70. Geburtstag, Innsbruck 2012, S. 48-56. L A T o u R, Bruno: Das Parlament der Dinge. Für eine politische Ökologie, Frankfurt am Main 2001. • "Drawing things together: Die Macht der unveränderlich mobilen Elemente«, in: Andréa Belliger / David J. Krieger (Hg.): ANThology. Ein einführendes Handbuch zur Akteur-Netzwerk-Theorie, Bielefeld 2006, S. 259-307. L E B o u R H I S, Jean-Pierre: „Quadriller le territoire. La cartographie au service de l'action publique contre les risques naturels«, in: Olivier Ihl / Martine Kaluszynski / Gilles Pollet (Hg.): Les sciences de gouvernement, Paris 2003, S. 157-167. L E F E B V R E, Henri: La production de l'espace, Paris 1974. L E H N E R T, Katrin / V O G E L, Lutz: „Einleitung. Kleinräumige Mobilität und Grenzwahrnehmung im 19. Jahrhundert", in: Katrin Lehnert / Lutz Vogel (Hg.): Transregionale Perspektiven. Kleinräumige Mobilität und Grenzwahrnehmung im 19. Jahrhundert, Dresden 2011, S. 9-22. L ö w , Martina: Raumsoziologie, Frankfurt am Main 2001. L U н м A N N , Niklas: Legitimation durch Verfahren, Frankfurt am Main 1983. M A I E R, Charles S.: »Leviathan 2.0: Inventing Modern Statehood«, in: Emily S. Rosenberg (Hg.): A World Connecting 1870-1945, Cambridge (Massachusetts) 2012, S. 27-282, 1000-1019. M A N N, Michael: The Sources of Social Power, 3 Bde., Cambridge 1986-2012. M A R I N E T T o, Michael: Social Theory, the State and Modern Society: The State in Contemporary Social Thought, Maidenhead 2007. M A R X, Leo: »Technology: The Emergence of a Hazardous Concept«, in: Technology and Culture. The International Quarterly of the Society for the History of Technology 51 (2010), S. 561-577. M A T H I E U, Jon: Die Alpen. Raum - Kultur - Geschichte, Stuttgart 2015. M c N E E LY, Ian F.: The Emancipation of Writing. German Civil Society in the Making 1790s-1820s, Berkeley 2003. M E J S T R I K, Alexander: „Raumvorstellungen in den Geschichts-, Sozial- 
und Kulturwissenschaften. Epistemologische Profile«, in: Alexander C. T. Geppert / Uffa Jensen / Jörn Weinhold (Hg.): Ortsgespräche. Raum und Kommunikation im 19. und 20. Jahrhundert, Bielefeld 2005, S. 53-77. M E U M A N N, Markus / P R Ö VE, Ralf: „Die Faszination des Staates und die historische Praxis. Zur Beschreibung von Herrschaftsbeziehungen jenseits teleologischer und dualistischer Begriffsbildungen«, in: Markus Meumann / Ralf Pröve (Hg.): Herrschaft in der Frühen Neuzeit. Umrisse eines dynamisch-kommunikativen Prozesses, Münster 2004, S. 11-49. M I G G E L B R I N K, Judith: „Die (Un-)Ordnung des Raumes. Bemerkungen zum Wandel geographischer Raumkonzepte im ausgehenden 20. Jahrhundert «, in: Alexander C. T. Geppert / Uffa Jensen / Jörn Weinhold (Hg.): Ortsgespräche. Raum und Kommunikation im 19. und 20. Jahrhundert, Bielefeld 2005, S. 79-105. M I L L E R, Peter / R o S E, Nikolas: Governing the Present. Administering Economic, Social and Personal Life, Cambridge 2008. M I т C HELL, Timothy: "The Limits of the State: Beyond Statist Approaches and Their Critics«, in: American Political Science Review 85 (1991), S. 77-96. M o L н о, Anthony: »Lo Stato e la finanza pubblica. Un'ipotesi basata sulla storia tardomedioevale di Firenze«, in: Giorgio Chittolini / Anthony Molho / Pierangelo Schiera (Hg.): Origini dello Stato. Processi di formazione statale in Italia fra medioevo ed età moderna, Bologna 1994, S. 225-280. M o N R o, Alexander: The Paper Trail. An Unexpected History of the World's Greatest Invention, London 2014. M u k E RJ I, Chandra: "The Unintended State«, in: Anthony Bennett / Patrick Joyce (Hg.): Material Powers. Cultural Studies, History and the Material Turn, London 2010, S. 81-101. M ü L L E R, Lothar: Weiße Magie. Die Epoche des Papiers, München 2012. N E T T L, John Peter: »The State as a Conceptual Variable«, in: World Politics. A Quarterly Journal of International Relations 20 (1967/68), S. 559-592. N I E U WE N B U R G, Paul / R U T G E R S, Mark R.: »Politics and Administration: Some Remarks on the Conceptual Roots of the Dichotomy«, in: Jahrbuch für europäische Verwaltungsgeschichte 12 (2000), S. 185-202. N I S B E T, Robert A.: The Sociological Tradition, New York 1966. O G B O R N , Miles: Indian Ink. Script and Print in the Making of the English East India Company, Chicago 2007. O S T E R H A M M E L, Jürgen: Die Verwandlung der Welt. Eine Geschichte des 19. Jahrhunderts, München 2009. O s т E R K A M P , Jana: „Wasser, Erde, Imperium. Eine kleine Politikgeschichte der Meliorationen in der Habsburgermonarchie«, in: Jörg Ganzenmüller / Tatjana Tönsmeyer (Hg.): Vom Vorrücken des Staates in die Fläche. Ein europäisches Phänomen des langen 19. Jahrhunderts, Köln 2016, S. 179-197. P A E T Z, Stephan: »Bürokratie«, in: Hans-Peter Müller / Steffen Sigmund (Hg.): Max Weber Handbuch. Leben - Werk - Wirkung, Stuttgart 2014, S. 38-41. P A L S K Y, Gilles: Des chiffres et des cartes. Naissance et développement de la cartographie quantitative française au XIXe siècle, Paris 1996. P A T R I A R C A, Silvana: Numbers and Nationhood. Writing Statistics in Nineteenth-Century Italy, Cambridge 1996. P I E R S O N , Christopher: The Modern State, London 1996. P O L L E Y, Rainer: »Kollegialprinzip und Geschäftsgang im 19. Jahrhundert. Eine verfassungs- und verwaltungsgeschichtliche Fallstudie zur Aktenkunde«, in: Archiv für Diplomatik, Schriftgeschichte, Siegelund Wappenkunde 42 (1996), S. 445-488. P о т , Andreas / B U DKE, Alexandra / K A N W I S C H E R, Detlef: »Internet, Raum und Gesellschaft. Zur Untersuchung eines dynamischen Verhältnisses«, in: Alexandra Budke / Detlef Kanwischer / Andreas Pott (Hg.): Internetgeographien. Beobachtungen zum Verhältnis von Internet, Raum und Gesellschaft, Stuttgart 2004, S. 9-20. R A N D E R I A, Shalini: "The State of Globalization. Legal Plurality, Overlapping Sovereignties and Ambiguous Alliances Between Civil Society and the Cunning State in India«, in: Theory, Culture and Society. Explorations in Critical Social Science 24/1 (2007), S. 1-33. RA o, Ursula / GREENLEAF, Graham William: "Subverting ID from Above and Below: The Uncertain Shaping of India's New Instrument of e-Governance«, in: Surveillance and Society 11 (2013), S. 287-300. R A P H A E L, Lutz: Recht und Ordnung. Herrschaft durch Verwaltung im
19. Jahrhundert, Frankfurt am Main 2000. • "Staat im Dorf. Transformation lokaler Herrschaft zwischen 1750 und 1850: Französische und westdeutsche Erfahrungen in vergleichender Perspektive«, in: Zeitschrift für Agrargeschichte und Agrarsoziologie 51 (2003), S. 43-61. R A U, Susanne: Räume. Konzepte, Wahrnehmungen, Nutzungen, Frankfurt am Main 2013. R E I N H A R D, Wolfgang: »Frühmoderner Staat - moderner Staat«, in: Olaf Mörke / Michael North (Hg.): Die Entstehung des modernen Europa 1600-1900, Köln 1998, S. 1-9. • Geschichte der Staatsgewalt. Eine vergleichende Verfassungsgeschichte Europas von den Anfängen bis zur Gegenwart, München 1999. • "Einführung: Moderne Staatsbildung eine ansteckende Krankheit?«, in: Wolfgang Reinhard / Elisabeth MüllerLuckner (Hg.): Verstaatlichung der Welt? Europäische Staatsmodelle und außereuropäische Machtprozesse, München 1999, S. VII-XIV. • »Geschichte der Staatsgewalt und europäische Expansion«, in: Wolfgang Reinhard / Elisabeth Müller-Luckner (Hg.): Verstaatlichung der Welt? Europäische Staatsmodelle und außereuropäische Machtprozesse, München 1999, S. 317-356. • »Zusammenfassung: Staatsbildung durch `Aushandeln«?«, in: Ronald G. Asch / Dagmar Freist (Hg.): Staatsbildung als kultureller Prozess. Strukturwandel und Legitimation von Herrschaft in der Frühen Neuzeit, Köln 2005, S. 429-438. • „No Statebuilding from Below! A Critical Commentary«, in: Willem Pieter Blockmans et al. (Hg.): Empowering Interactions. Political Cultures and the Emergence of the State in Europe 1300-1900, Farnham 2009, S. 299-304. R E Y N O L D S, Susan: "The Historiography of the Medieval State«, in: Michael Bentley (Hg.): Companion to Historiography, London 1997, S. 117-138. R u t z, Andreas: „Grenzüberschreitungen im deutsch-niederländisch-französischen Grenzraum«, in: Christine Roll / Frank Pohle / Matthias Myrczek (Hg.): Grenzen und Grenzüberschreitungen. Bilanz und Perspektiven der Frühneuzeitforschung, Köln 2010, S. 217-222. S A C K, Robert David: Human Territoriality. Its Theory and History, Cambridge 1986. S C н A R T E, Sebastian: „Vom Bestreben, auch an den äußersten Westmarken nach Kräften der vaterländischen Sache zu dienen. Die rheinpreußischen Grenzkreise Eupen und Malmedy 1815-1914«, in: Katrin Lehnert / Lutz Vogel (Hg.): Transregionale Perspektiven. Kleinräumige Mobilität und Grenzwahrnehmung im 19. Jahrhundert, Dresden 2011, S. 191-203. S C H E D LER, Kuno / P R O E L L E R, Isabella: New Public Management, Bern ${ }^{5} 2011$. S C H L ö G L, Rudolf: Anwesende und Abwesende. Grundriss für eine Gesellschaftsgeschichte der Frühen Neuzeit, Konstanz 2014. S C H M I T Z - B E R N I N G, Cornelia: Vokabular des Nationalsozialismus, Berlin 1998. S C H N E I D E R, Michael C.: Wissensproduktion im Staat. Das königlich preußische statistische Bureau 1860-1914, Frankfurt am Main 2013. S c H R O E R, Markus: Räume, Orte, Grenzen. Auf dem Weg zu einer Soziologie des Raums, Frankfurt am Main 2006. S с о т т, James C.: Seeing Like a State. How Certain Schemes to Improve the Human Condition Have Failed, New Haven 1998. S E I B E L, Wolfgang: "Studying Hybrids: Sectors and Mechanisms", in: Organization Studies. An International Multidisciplinary Journal Devoted to the Study of Organizations, Organizing, and the Organized in and between Societies 36 (2015), S. 697-712. • Verwaltung verstehen. Eine theoriegeschichtliche Einführung, Frankfurt am Main 2016. S I E G E R T, Bernhard: Passage des Digitalen. Zeichenpraktiken der neuzeitlichen Wissenschaften 1500-1900, Berlin 2003. • / V o G L, Joseph (Hg.): Europa. Kultur der Sekretäre, Zürich 2003. S к O R N I C K I, Arnault: „Constructions pratiques et symboliques des frontières politiques«, in: Antonin Cohen / Bernard Lacroix / Philippe Riutort (Hg.): Nouveau manuel de science politique, Paris 2009, S. 74-85. S P I E K E R, Sven (Hg.): Bürokratische Leidenschaften. Kultur- und Mediengeschichte im Archiv, Berlin 2004. S т E I N M E T Z, George: "Introduction: Culture and the State«, in: George Steinmetz ( $\mathrm{Hg}$.): State/Culture. State-Formation after the Cultural Turn, Ithaca 1999, S. 1-49. S T o L L B E R G - R I L I N G E R, Barbara: »Zeremoniell, Ritual, Symbol. Neue Forschungen zur symbolischen Kommunikation in Spätmittelalter und Früher Neuzeit«, in: Zeitschrift für Historische 
Forschung 27 (2000), S. 389-405. S T O L LE I S, Michael: Geschichte des öffentlichen Rechts in Deutschland, Bd. 2: Staatsrechtslehre und Verwaltungswissenschaft 1800-1914, München 1992. T A N T N E R, Anton: Ordnung der Häuser, Beschreibung der Seelen. Hausnummerierung und Seelenkonskription in der Habsburgermonarchie, Innsbruck 2007. T E U S C H ER, Simon: Erzähltes Recht. Lokale Herrschaft, Verschriftlichung und Traditionsbildung im Spätmittelalter, Frankfurt am Main 2007. V I E R H A U S, Rudolf: „The Prussian Bureaucracy Reconsidered«, in: John Brewer / Eckhart Hellmuth (Hg.): Rethinking Leviathan. The Eighteenth-Century State in Britain and Germany, Oxford 1999, S. 149-165. V I S M A N N , Cornelia: Akten. Medientechnik und Recht, Frankfurt am Main 2000. V o I G T, Rüdiger: Den Staat denken. Der Leviathan im Zeichen der Krise, Baden-Baden 2007. W e в E R, Max: Geistige Arbeit als Beruf. Vier Vorträge vor dem Freistudentischen Bund, Bd. 2: Politik als Beruf, München 1919. - Wirtschaft und Gesellschaft. Grundriss der verstehenden Soziologie. Studienausgabe, hg. von Johannes Winckelmann, Tübingen ${ }^{5} 1976$. W E I c H H A R D T, Peter: »Territorialität, Identität und Grenzerfahrung", in: Peter Haslinger (Hg.): Grenze im Kopf. Beiträge zur Geschichte der Grenze in Ostmitteleuropa, Frankfurt am Main 1999, S. 19-30. W E R L E, Dirk: Copia librorum. Problemgeschichte imaginierter Bibliotheken 15801630, Tübingen 2007. W E R L E N , Benno: »Körper, Raum und mediale Repräsentation«, in: Jörg Döring / Tristan Thielmann (Hg.): Spatial Turn. Das Raumparadigma in den Kultur- und Sozialwissenschaften, Bielefeld 2008, S. 365-392. W U N D E R, Bernd: Privilegierung und Disziplinierung. Die Entstehung des Berufsbeamtentums in Bayern und Württemberg (1780-1825), München 1978. Y A t E S, JoAnne: Control through Communication. The Rise of System in American Management, Baltimore 1989. 
1 Georg Meyer: Lehrbuch des deutschen Staatsrechtes, Leipzig 1878, S. 4: "Der Staat ist ein auf ein bestimmtes Gebiet basirtes menschliches Gemeinwesen. Die Grundlagen desselben sind daher: 1. eine Menge von Menschen als persönliche Grundlage. [...] 2. ein Land oder Gebiet als sachliche Grundlage«. Zu den Entstehungskontexten dieser Lehre vgl. Thomas Ellwein: Der Staat als Zufall und als Notwendigkeit. Die jüngere Verwaltungsentwicklung in Deutschland am Beispiel Ostwestfalen-Lippe, 2 Bde., Opladen 1993-1997, Bd. 1, S. 28-29.

2 Max Weber: Geistige Arbeit als Beruf. Vier Vorträge vor dem Freistudentischen Bund, Bd. 2: Politik als Beruf, München 1919, S. 4: "Staat ist diejenige menschliche Gemeinschaft, welche innerhalb eines bestimmten Gebietes - dies: das >Gebietı, gehört zum Merkmal - das monopol legitimer physischer Gewaltsamkeit für sich (mit Erfolg) beansprucht«. Zur Verwandtschaft jener Definition mit der staatsrechtlichen vgl. Stefan Breuer: Der Staat. Entstehung, Typen, Organisationsstadien, Reinbek bei Hamburg 1998, S. 18.

3 Beispielsweise Charles S. Maier: "Leviathan 2.0: Inventing Modern Statehood", in: Emily S. Rosenberg (Hg.): A World Connecting 18701945, Cambridge (Massachusetts) 2012, S. 27-282, 1000-1019, hier S. 33-34, 1001; Susan Reynolds: "The Historiography of the Medieval State«, in: Michael Bentley (Hg.): Companion to Historiography, London 1997, S. 117-138, hier S. 118; Michael Mann: The Sources of Social Power, 3 Bde., Cambridge 1986-2012, Bd. 1, S. 37; Bd. 2, S. 55.

4 Patrick Joyce: The State of Freedom: A Social History of the British State since 1800, Cambridge 2013, S. 59: "The post enabled the incorporation of the disparate territories and subjects of the kingdom into something like a unit (albeit often a fluid one)".

5 Joyce: State of Freedom, S. 20-21, 23-24, 51-184.

6 Explizit ist die Einstufung als trivial etwa bei Michael Bentley: ")Boundaries in Theoretical Language about the British State«, in: Simon J. D. Green / Richard C. Whiting (Hg.): The Boundaries of the State in Modern Britain, Cambridge 1996, S. 29-56, hier S. 29-30. Es finden sich auch umfangreiche Werke über die Theorie des Staates, in denen der räumliche Aspekt nur beiläufig erwähnt, aber nirgends thematisiert wird, etwa Andreas Anter: Max Webers Theorie des modernen Staates. Herkunft, Struktur und Bedeutung, Berlin 1995. Wo dies doch geschieht, wird der Anfang nicht selten mit der Zurückweisung der scheinbaren Selbstverständlichkeit gemacht, etwa bei Christopher Pierson: The Modern State, London 1996, S. 12.

7 Der Ausdruck meint hier im Sinne von James C. Scott jene Zeitspanne zwischen dem ausgehenden 19. und dem dritten Viertel des 20. Jh., in welcher der Glaube an einen linearen >Fortschritt، samt der Bereitschaft zu großflächigen und invasiven Interventionen zu seiner Durchsetzung - als deren Träger zuvorderst der Staat auftrat - seinen Höhepunkt erreichte; vgl. James C. Scott: Seeing Like a State. How Certain Schemes to Improve the Human Condition Have Failed, New Haven 1998, S. 88-90.

8 Lutz Raphael: Recht und Ordnung. Herrschaft durch Verwaltung im 19. Jahrhundert, Frankfurt am Main 2000, S. 10-15.

9 Maier, »Leviathan 2.0«, S. 148-152, 156-170; Jürgen Osterhammel: Die Verwandlung der Welt. Eine Geschichte des 19. Jahrhunderts, München 2009, S. 866-882; Christopher A. Bayly: The Birth of the Modern World 1780-1914. Global Connections and Comparisons, Malden 2004, S. 247-283; Wolfgang Reinhard: "Geschichte der Staatsgewalt und europäische Expansion«, in: Wolfgang Reinhard / Elisabeth Müller-Luckner (Hg.): Verstaatlichung der Welt? Europäische Staatsmodelle und außereuropäische Machtprozesse, München 1999, S. 317-356, hier S. 328-345.

10 Vgl. Wolfgang Reinhard: Geschichte der Staatsgewalt. Eine vergleichende Verfassungsgeschichte Europas von den Anfängen bis zur Gegenwart, München 1999, S. 16.
11 Neuere Einstiege und Überblicke bieten etwa Michael Marinetto: Social Theory, the State and Modern Society: The State in Contemporary Social Thought, Maidenhead 2007; Rüdiger Voigt: Den Staat denken. Der Leviathan im Zeichen der Krise, Baden-Baden 2007; Jens Bartelson: The Critique of the State, Cambridge 2001; Arthur Benz: Der moderne Staat. Grundlagen der politologischen Analyse, München 2001, S. 9-74.

12 Dazu einführend Susanne Rau: Räume. Konzepte, Wahrnehmungen, Nutzungen, Frankfurt am Main 2013; Markus Schroer: Räume, Orte, Grenzen. Auf dem Weg zu einer Soziologie des Raums, Frankfurt am Main 2006; Martina Löw: Raumsoziologie, Frankfurt am Main 2001.

13 Horst Bredekamp: Thomas Hobbes visuelle Strategien. Der Leviathan: Urbild des modernen Staates. Werkillustrationen und Portraits, Berlin 1999, S. 15-16, 79-82; vgl. Voigt: Den Staat denken, S. 233-234; Oleg Kharkhordin: "What Is the State? The Russian Concept of Gosudarstvo in the European Context«, in: History and Theory. Studies in the Philosophy of History 40 (2001), S. 206-240, hier S. 239.

14 Vgl. Lutz Danneberg: Die Anatomie des Text-Körpers und Natur-Körpers. Das Lesen im liber naturalis und supernaturalis, Berlin 2003, S. 46.

15 Joyce: State of Freedom, S. 14.

16 Bernard Lacroix: „Ordre politique et ordre social. Objectivisme, objectivation et analyse politique«, in: Madeleine Grawitz / Jean Leca (Hg.): Traité de science politique, Bd. 1: La science politique, science sociale. L'ordre politique, Paris 1985, S. 469-565, hier S. 476-477.

17 Vgl. Kharkhordin: „What Is the State«, S. 206-207; Michael J. Braddick: State Formation in Early Modern England c. 1550-1700, Cambridge 2000, S. 90-91. Zur Hinderlichkeit vieler verbreiteter Sprachgewohnheiten in Bezug auf den Staat, deren Banalisierung die hochintentionalen Motive ihrer Entstehung vergessen lässt, vgl. Bernard Lacroix: „Genèses et constructions de l'État moderne«, in: Antonin Cohen / Bernard Lacroix / Philippe Riutort (Hg.): Nouveau manuel de science politique, Paris 2009, S. 52-70, hier S. 52.

18 Bartelson: Critique, S. 12-13; Lacroix: "Ordre politique«, S. 475-476.

19 Mann: Sources of Social Power, Bd. 2, S. 61.

20 Bentley: "Boundaries«.

21 Timothy Mitchell: „The Limits of the State: Beyond Statist Approaches and Their Critics«, in: American Political Science Review 85 (1991), S. 77-96, hier S. 90. Ein drastisches Beispiel bieten die von Tolga Esmer eindringlich beschriebenen Taktiken des osmanischen Hofes um 1800, Anführer irregulärer Streitkräfte auf der Balkanhalbinsel je nach Situation als Banditen zu bekämpfen oder als Würdenträger zu kooptieren; vgl. Tolga U. Esmer: »Economies of Violence, Banditry and Governance in the Ottoman Empire around 1800 «, in: Past and Present. A Journal of Historical Studies 224 (2014), S. 163-199.

22 Lutz Raphael: "Staat im Dorf. Transformation lokaler Herrschaft zwischen 1750 und 1850: Französische und westdeutsche Erfahrungen in vergleichender Perspektive«, in: Zeitschrift für Agrargeschichte und Agrarsoziologie 51 (2003), S. 43-61, hier S. 58-60.

23 Zu diesem Begriff vgl. Raphael: Recht und Ordnung, S. 23.

24 Stephan Paetz: „Bürokratie«, in: Hans-Peter Müller / Steffen Sigmund (Hg.): Max Weber Handbuch. Leben - Werk - Wirkung, Stuttgart 2014, S. 38-41, hier S. 39-40; Anter: Max Webers Theorie, S. 172-184. Zur Kritik in den jüngeren Debatten etwa André Holenstein: »Introduction: Empowering Interactions: Looking at Statebuilding from Below«, in: Willem Pieter Blockmans et al. (Hg.): Empowering Interactions. Political Cultures and the Emergence of the State in Europe 1300-1900, Farnham 2009, S. 1-31, hier S. 7, der von »the Weberian trap « spricht; Rudolf Vierhaus: "The Prussian Bureaucracy 
Reconsidered«, in: John Brewer / Eckhart Hellmuth (Hg.): Rethinking Leviathan. The Eighteenth-Century State in Britain and Germany, Oxford 1999, S. 149-165, hier S. 149-152; Anthony Molho: "Lo Stato e la finanza pubblica. Un'ipotesi basata sulla storia tardomedioevale di Firenze«, in: Giorgio Chittolini / Anthony Molho / Pierangelo Schiera (Hg.): Origini dello Stato. Processi di formazione statale in Italia fra medioevo ed età moderna, Bologna 1994, S. 225-280, hier S. 230-231.

$25 \mathrm{Zu}$ Kontinuitäten zwischen Tocqueville und Weber vgl. Richard Swedberg: "Tocqueville as a Pioneer in Organization Theory«, in: Paul S. Adler (Hg.): The Oxford Handbook of Sociology and Organization Studies. Classical Foundations, Oxford 2009, S. 39-61, hier S. 52-54; Robert A. Nisbet: The Sociological Tradition, New York 1966, S. 141, 144-148.

26 Bayly: Birth of the Modern World, S. 252-253.

27 Übersichten bieten etwa Jörg Ganzenmüller / Tatjana Tönsmeyer: "Einleitung: Vom Vorrücken des Staates in die Fläche. Ein europäisches Phänomen des langen 19. Jahrhunderts«, in: Jörg Ganzenmüller / Tatjana Tönsmeyer (Hg.): Vom Vorrücken des Staates in die Fläche. Ein europäisches Phänomen des langen 19. Jahrhunderts, Köln 2016, S. 7-31; Holenstein: „Empowering Interactions«; Dagmar Freist: „Einleitung: Staatsbildung, lokale Herrschaftsprozesse und kultureller Wandel in der Frühen Neuzeit«, in: Ronald G. Asch / Dagmar Freist (Hg.): Staatsbildung als kultureller Prozess. Strukturwandel und Legitimation von Herrschaft in der Frühen Neuzeit, Köln 2005, S. 1-47; Markus Meumann / Ralf Pröve: „Die Faszination des Staates und die historische Praxis. Zur Beschreibung von Herrschaftsbeziehungen jenseits teleologischer und dualistischer Begriffsbildungen«, in: Markus Meumann / Ralf Pröve (Hg.): Herrschaft in der Frühen Neuzeit. Umrisse eines dynamisch-kommunikativen Prozesses, Münster 2004, S. 11-49.

28 Holenstein: »Empowering Interactions«, S. 4-5.

29 Niels Grüne: "Local Demand for Order and Government Intervention: Social Group Conflicts as Statebuilding Factors in Villages of the Rhine Palatinate, c. 1760-1810«, in: Willem Pieter Blockmans et al. (Hg.): Empowering Interactions. Political Cultures and the Emergence of the State in Europe 1300-1900, Farnham 2009, S. 173-186; Martin Dinges: "Justiznutzungen als soziale Kontrolle in der Frühen Neuzeit«, in: Andreas Blauert / Gerd Schwerhoff (Hg.): Kriminalitätsgeschichte. Beiträge zur Sozial- und Kulturgeschichte der Vormoderne, Konstanz 2000, S. 503-544.

30 André Holenstein: »Kommunikatives Handeln im Umgang mit Policeyordnungen. Die Markgrafschaft Baden im 18. Jahrhundert«, in: Ronald G. Asch / Dagmar Freist (Hg.): Staatsbildung als kultureller Prozess. Strukturwandel und Legitimation von Herrschaft in der Frühen Neuzeit, Köln 2005, S. 191-208, hier S. 197-204.

31 Wolfgang Reinhard: "Zusammenfassung: Staatsbildung durch sAushandeln«?», in: Ronald G. Asch / Dagmar Freist (Hg.): Staatsbildung als kultureller Prozess. Strukturwandel und Legitimation von Herrschaft in der Frühen Neuzeit, Köln 2005, S. 429-438, hier S. 430.

32 Martin Dinges: "Aushandeln von Armut in der Frühen Neuzeit: Selbsthilfepotential, Bürgervorstellungen und Verwaltungslogiken«, in: WerkstattGeschichte 10 (1995), S. 7-15; vgl. Meumann / Pröve: »Faszination«, S. 43-44.

33 Beides bei Michael J. Braddick: "State Formation and Political Culture in Elizabethan and Stuart England. Micro-histories and Macro-historical Change«, in: Ronald G. Asch / Dagmar Freist (Hg.): Staatsbildung als kultureller Prozess. Strukturwandel und Legitimation von Herrschaft in der Frühen Neuzeit, Köln 2005, S. 69-90, hier S. 70.

34 Wolfgang Reinhard: "No Statebuilding from Below! A Critical Commentary«, in: Willem Pieter Blockmans et al. (Hg.): Empowering
Interactions. Political Cultures and the Emergence of the State in Europe 1300-1900, Farnham 2009, S. 299-304.

35 Pierre Bourdieu: Sur l'État. Cours au Collège de France (1989-1992), hg. von Patrick Champagne et al., Paris 2012, S. 196-199; Wolfgang Reinhard: »Frühmoderner Staat - moderner Staat«, in: Olaf Mörke / Michael North (Hg.): Die Entstehung des modernen Europa 16001900, Köln 1998, S. 1-9, hier S. 1, 9.

36 Dies ist auch bereits ausdrücklich festgehalten bei Dinges: „Aushandeln von Armut«, S. 7; außerdem vgl. Ute Frevert: "Neue Politikgeschichte«, in: Joachim Eibach / Günther Lottes (Hg.): Kompass der Geschichtswissenschaft. Ein Handbuch, Göttingen 2002, S. 152-164, hier S. 158.

37 Sabine Holtz: "Wissen und Macht. Territorialstaat und Bildungspolitik im 17. Jahrhundert«, in: Historisches Jahrbuch 123 (2003), S. 179-196, hier S. 179.

38 Holenstein: »Empowering Interactions«, S. 5; vgl. Joyce: State of Freedom, S. 22.

39 Joyce: State of Freedom, S. 19. Joyce verwendet hier auch den verbreiteteren Begriff des Netzwerks (»network«); jener der Anordnung hat allerdings den Vorteil, dass er nicht nur die Verbundenheit, sondern auch die relative Position einzelner Elemente als signifikant anspricht. Eine ähnliche Perspektive auf den Staat unter expliziter Berufung auf die Actor-Network-Theorie nach Bruno Latour bietet Margareth Lanzinger: "Das Lokale neu positionieren im actor-network-Raum - globalgeschichtliche Herausforderungen und illyrische Steuerpolitiken«, in: Ewald Hiebl / Ernst Langthaler (Hg.): Im Kleinen das Große suchen. Mikrogeschichte in Theorie und Praxis. Hanns Haas zum 70. Geburtstag, Innsbruck 2012, S. 48-56.

40 Manuel DeLanda: A New Philosophy of Society. Assemblage Theory and Social Complexity, London 2006, S. 3-5.

41 DeLanda: New Philosophy, S. 12.

42 Zu diesem Konzept vgl. Peter Becker / William Clark: »Introduction«, in: Peter Becker / William Clark (Hg.): Little Tools of Knowledge. Historical Essays on Academic and Bureaucratic Practices, Ann Arbor 2001, S. 1-34.

43 Anter: Max Webers Theorie, S. 24-35; vgl. Catherine Brennan: Max Weber on Power and Social Stratification. An Interpretation and Critique, Aldershot 1997, S. 219.

44 Breuer: Staat, S. 19; Pierson: Modern State, S. 9-11; Mann: Sources of Social Power, Bd. 2, S. 55.

45 Vgl. Anter: Max Webers Theorie, S. 35-47.

46 Bourdieu: Sur l'État, S. 311-347; vgl. Lacroix: „Genèses«, S. 54-60.

47 Benz: Staat, S. 74.

48 John Peter Nettl: »The State as a Conceptual Variable«, in: World Politics. A Quarterly Journal of International Relations 20 (1967/68), S. 559592, hier S. 566; vgl. Reinhard: Geschichte der Staatsgewalt, S. 18-19.

49 Joyce: State of Freedom, S. 14; Kharkhordin: "What Is the State», S. 209; George Steinmetz: »Introduction: Culture and the State», in: George Steinmetz (Hg.): State/Culture. State-Formation after the Cultural Turn, Ithaca 1999, S. 1-49, hier S. 27.

50 Joyce: State of Freedom, S. 30.

51 Leo Marx: „Technology: The Emergence of a Hazardous Concept«, in: Technology and Culture. The International Quarterly of the Society for the History of Technology 51 (2010), S. 561-577, hier S. 567-572.

52 Chandra Mukerji: "The Unintended State«, in: Anthony Bennett / Patrick Joyce (Hg.): Material Powers. Cultural Studies, History and the Material Turn, London 2010, S. 81-101.

53 Osterhammel: Verwandlung, S. 1012-1029; Alexander C. T. Geppert / Uffa Jensen / Jörn Weinhold: „Verräumlichung. Kommunikative Praktiken in historischer Perspektive, 1840-1930«, in: Alexander C. T. Geppert / Uffa Jensen / Jörn Weinhold (Hg.): Ortsgespräche. Raum und 
Kommunikation im 19. und 20. Jahrhundert, Bielefeld 2005, S. 15-49, hier S. 29-43; Raphael: Recht und Ordnung, S. 14-15.

54 Jana Osterkamp: „Wasser, Erde, Imperium. Eine kleine Politikgeschichte der Meliorationen in der Habsburgermonarchie«, in: Jörg Ganzenmüller / Tatjana Tönsmeyer (Hg.): Vom Vorrücken des Staates in die Fläche. Ein europäisches Phänomen des langen 19. Jahrhunderts, Köln 2016, S. 179-197.

55 Jonathan Agar: The Government Machine. A Revolutionary History of the Computer, Cambridge (Massachusetts) 2003, S. 45-142.

56 Zur Bedeutung von Schrift für die Anfänge des Staates vgl. Michael Clanchy: »Does Writing Construct the State?«, in: The Journal of Historical Sociology 15 (2002), S. 68-70. Untersuchungen von Verwaltung im 19. Jahrhundert unter dem Blickwinkel der Schriftlichkeit bieten etwa Miles Ogborn: Indian Ink. Script and Print in the Making of the English East India Company, Chicago 2007; Ian F. McNeely: The Emancipation of Writing. German Civil Society in the Making 1790s - 1820s, Berkeley 2003.

57 Lothar Müller: Weiße Magie. Die Epoche des Papiers, München 2012, S. 56-64; kurz auch Alexander Monro: The Paper Trail. An Unexpected History of the World's Greatest Invention, London 2014, S. 291.

58 Joyce: State of Freedom, S. 33-50.

59 Sandro-Angelo Fusco et al.: »Verwaltung, Amt, Beamter«, in: Otto Brunner / Werner Conze / Reinhart Koselleck (Hg.): Geschichtliche Grundbegriffe. Historisches Lexikon der politisch-sozialen Sprache in Deutschland, Bd. 7, Stuttgart 1992, S. 1-96, hier S. 5.

60 Johann Heinrich Gottlob von Justi: Grundsätze der Policeywissenschaft in einem vernünftigen, auf den Endzweck der Policey gegründeten, Zusammenhange zum Gebrauch Academischer Vorlesungen abgefasset, Göttingen ${ }^{3} 1782$, S. 4.

61 Exemplarisch vgl. Günther Heinrich von Berg: Handbuch des Teutschen Policeyrechts, Teil 1, Hannover ${ }^{21802, ~ S . ~} 12$.

62 Charles-Louis de Secondat de Montesquieu: De I'Esprit des lois, Bd. 1, London 1777, S. 311.

63 Beispielsweise Montesquieu: De l'Esprit des lois, Bd. 1, S. 325-326.

64 Wulf Damkowski: Die Entstehung des Verwaltungsbegriffes. Eine Wortstudie, Köln 1969, S. 187.

65 Exemplarisch vgl. Jacques Chevallier: Science administrative, Paris 32002, S. 15-19; Michael Stolleis: Geschichte des öffentlichen Rechts in Deutschland, Bd. 2: Staatsrechtslehre und Verwaltungswissenschaft 1800-1914, München 1992; Ferrel Heady: Public Administration: A Comparative Perspective, New York ${ }^{5} 1996$.

66 Dazu kanonisch Woodrow Wilson: "The Study of Administration«, in: Political Science Quarterly 2 (1887), S. 198-222; und historisch Paul Nieuwenburg / Mark R. Rutgers: "Politics and Administration: Some Remarks on the Conceptual Roots of the Dichotomy«, in: Jahrbuch für europäische Verwaltungsgeschichte 12 (2000), S. 185-202.

67 Ein kurzer Überblick zur älteren Verfassungsgeschichte findet sich bei Joachim Eibach: „Verfassungsgeschichte als Verwaltungsgeschichte», in: Joachim Eibach / Günther Lottes (Hg.): Kompass der Geschichtswissenschaft, Göttingen 2002, S. 142-151, hier S. 144-146.

68 Max Weber: Wirtschaft und Gesellschaft. Grundriss der verstehenden Soziologie. Studienausgabe, hg. von Johannes Winckelmann, Tübingen ${ }^{5} 1976$, S. $124-130$.

69 Exemplarisch vgl. Bernd Wunder: Privilegierung und Disziplinierung. Die Entstehung des Berufsbeamtentums in Bayern und Württemberg (1780-1825), München 1978; Jürgen Kocka: Unternehmensverwaltung und Angestelltenschaft am Beispiel Siemens 1847-1914. Zum Verhältnis von Kapitalismus und Bürokratie in der deutschen Industrialisierung, Stuttgart 1969; Reinhart Koselleck: Preußen zwischen Reform und Revolution. Allgemeines Landrecht, Verwaltung und soziale Bewegung von 1791 bis 1848, Stuttgart 1967.
70 Kuno Schedler / Isabella Proeller: New Public Management, Bern 52011; Arthur Benz / Nicolai Dose (Hg.): Governance - Regieren in komplexen Regelsystemen. Eine Einführung, Wiesbaden ${ }^{2} 2010$.

71 Wolfgang Seibel: "Studying Hybrids: Sectors and Mechanisms», in: Organization Studies. An International Multidisciplinary Journal Devoted to the Study of Organizations, Organizing, and the Organized in and between Societies 36 (2015), S. 697-712; Ursula Rao / Graham William Greenleaf: "Subverting ID From Above and Below: The Uncertain Shaping of India's New Instrument of e-Governance«, in: Surveillance and Society 11 (2013), S. 287-300; Armin von Bogdandy / Philipp Dann: "International Composite Administration: Conceptualizing Multi-Level and Network Aspects in the Exercise of International Public Authority«, in: German Law Journal. Developments in German, European and International Jurisprudence 9 (2008), S. 2013-2039; Shalini Randeria: "The State of Globalization. Legal Plurality, Overlapping Sovereignties and Ambiguous Alliances Between Civil Society and the Cunning State in India", in: Theory, Culture and Society. Explorations in Critical Social Science 24/1 (2007), S. 1-33; Claudio Franzius: „Der >Gewährleistungsstaat - ein neues Leitbild für den sich wandelnden Staat?«, in: Der Staat. Zeitschrift für Staatslehre und Verfassungsgeschichte, deutsches und europäisches öffentliches Recht 42 (2003), S. 493-517.

72 Im Überblick vgl. Benjamin Kafka: »Paperwork: The State of the Discipline«, in: Book History 12 (2009), S. 340-353.

73 Cornelia Vismann: Akten. Medientechnik und Recht, Frankfurt am Main 2000.

74 Friedrich Balke / Bernhard Siegert / Joseph Vogl (Hg.): „Medien der Bürokratie», in: Archiv für Mediengeschichte 16 (2016); Sven Spieker (Hg.): Bürokratische Leidenschaften. Kultur- und Mediengeschichte im Archiv, Berlin 2004; Bernhard Siegert / Joseph Vogl (Hg.): Europa. Kultur der Sekretäre, Zürich 2003.

75 Michel Foucault: "Das Leben der infamen Menschen«, in: Michel Foucault: Schriften in vier Bänden. Dits et Ecrits, Bd. 3, hg. von Daniel Defert / François Ewald / Jacques Lagrange, Frankfurt am Main 2003, S. 309-332, hier S. 314.

76 Bernhard Siegert: Passage des Digitalen. Zeichenpraktiken der neuzeitlichen Wissenschaften 1500-1900, Berlin 2003, S. 25.

77 Lorenz Engell / Bernhard Siegert: »Editorial«, in: Zeitschrift für Medien- und Kulturforschung 1 (2010), S. 5-9, hier S. 7.

78 Dirk Baecker: „Durch diesen schönen Fehler mit sich selbst bekannt gemacht«, in: Dirk Baecker: Organisation und Management. Aufsätze, Frankfurt am Main 2003, S. 18-40, hier S. 30.

79 Dazu grundlegend Niklas Luhmann: Legitimation durch Verfahren, Frankfurt am Main 1983.

80 Bruno Latour: »Drawing things together: Die Macht der unveränderlich mobilen Elemente«, in: Andréa Belliger / David J. Krieger (Hg.): ANThology. Ein einführendes Handbuch zur Akteur-Netzwerk-Theorie, Bielefeld 2006, S. 259-307, hier S. 296.

81 Matthew S. Hull: Government of Paper. The Materiality of Bureaucracy in Urban Pakistan, Berkeley 2012.

82 Dazu u. a. Benjamin Kafka: The Demon of Writing. Powers and Failures of Paperwork, New York 2012; Delphine Gardey: Écrire, calculer, classer. Comment une révolution de papier a transformé les sociétés contemporaines (1800-1940), Paris 2008; Thomas G. Kirsch: Spirits and Letters. Reading, Writing and Charisma in African Christianity, New York 2008; Peter Miller / Nikolas Rose: Governing the Present. Administering Economic, Social and Personal Life, Cambridge 2008; Kenneth W. Abbott / Duncan Snidal: »Hard and Soft Law in International Governance«, in: Judith L. Goldstein et al. (Hg.): Legalization and World Politics, Cambridge (Massachusetts) 2001, S. 37-72; Margaret E. Keck / Kathryn Sikkink: Activists Beyond 
Borders. Advocacy Networks in International Politics, Ithaca 1998; JoAnne Yates: Control through Communication. The Rise of System in American Management, Baltimore 1989.

83 Wolfgang Seibel: Verwaltung verstehen. Eine theoriegeschichtliche Einführung, Frankfurt am Main 2016.

84 Exemplarisch vgl. Rudolf Schlögl: Anwesende und Abwesende. Grundriss für eine Gesellschaftsgeschichte der Frühen Neuzeit, Konstanz 2014; Willem Pieter Blockmans et al. (Hg.): Empowering Interactions. Political Cultures and the Emergence of the State in Europe 1300-1900, Farnham 2009; Simon Teuscher: Erzähltes Recht. Lokale Herrschaft, Verschriftlichung und Traditionsbildung im Spätmittelalter, Frankfurt am Main 2007; Hagen Keller: „Vom sheiligen Buch zur >Buchführung`. Lebensfunktionen der Schrift im Mittelalter«, in: Frühmittelalterliche Studien. Jahrbuch des Instituts für Frühmittelalterforschung der Universität Münster 26 (1992), S. 1-31; Harold A. Innis: Empire and Communications, Oxford 1950.

85 Johann Heinrich Zedler (Hg.): Grosses vollständiges UniversalLexicon aller Wissenschaften und Künste, Bd. 30, Leipzig 1741, Sp. 1793-1817, s. v. Regierung, Regiment, hier Sp. 1793.

86 Michel Foucault: Geschichte der Gouvernementalität, Bd. 1: Sicherheit, Territorium, Bevölkerung. Vorlesung am Collège de France 1977-78, hg. von Michel Senellart, Frankfurt am Main 2004, S. 506.

87 Entsprechend der berühmten Definition von Otto Mayer: Deutsches Verwaltungsrecht, Bd. 1, Leipzig 1895, S. 9.

88 Pascale Cancik: „Verwaltung, Raum, Verwaltungsraum - eine historische Annäherung«, in: Hermann Hill / Utz Schliesky (Hg.): Die Vermessung des virtuellen Raums. E-Volution des Rechts- und Verwaltungssystems III, Baden-Baden 2012, S. 29-54, hier S. 30-32.

89 Vgl. Barbara Stollberg-Rilinger: "Zeremoniell, Ritual, Symbol. Neue Forschungen zur symbolischen Kommunikation in Spätmittelalter und Früher Neuzeit«, in: Zeitschrift für Historische Forschung 27 (2000), S. 389-405, hier S. 396-397.

90 Ann M. Blair: Too Much to Know. Managing Scholarly Information before the Modern Age, New Haven 2010, S. 161-164; Dirk Werle: Copia librorum. Problemgeschichte imaginierter Bibliotheken 1580-1630, Tübingen 2007, S. 170-222.

91 Markus Friedrich: „Das Buch als Theater. Überlegungen zu Signifikanz und Dimensionen der Theatrum-Metapher als frühneuzeitlichem Buchtitel», in: Theodor Stammen et al. (Hg.): Wissenssicherung, Wissensordnung und Wissensverarbeitung. Das europäische Modell der Enzyklopädien, Berlin 2004, S. 205-232.

92 Dazu kurz Benno Werlen: „Körper, Raum und mediale Repräsentation«, in: Jörg Döring / Tristan Thielmann (Hg.): Spatial Turn. Das Raumparadigma in den Kultur- und Sozialwissenschaften, Bielefeld 2008, S. 365-392, hier S. 367-368; Cornelia Schmitz-Berning: Vokabular des Nationalsozialismus, Berlin 1998, S. 375-380; vgl. Geppert / Jensen / Weinhold: „Verräumlichung«, S. 21.

93 Alexander Mejstrik: »Raumvorstellungen in den Geschichts-, Sozialund Kulturwissenschaften. Epistemologische Profile«, in: Alexander C. T. Geppert / Uffa Jensen / Jörn Weinhold (Hg.): Ortsgespräche. Raum und Kommunikation im 19. und 20. Jahrhundert, Bielefeld 2005, S. 53-77.

94 Judith Miggelbrink: "Die (Un-)Ordnung des Raumes. Bemerkungen zum Wandel geographischer Raumkonzepte im ausgehenden 20. Jahrhundert«, in: Alexander C. T. Geppert / Uffa Jensen / Jörn Weinhold (Hg.): Ortsgespräche. Raum und Kommunikation im 19. und 20. Jahrhundert, Bielefeld 2005, S. 79-105, hier S. 87.

95 Cancik: „Verwaltung«, S. 53.

96 Löw: Raumsoziologie, S. 9.

97 Miggelbrink: "(Un-)Ordnung«, S. 83-84.

98 Als allgemeinen Einstieg vgl. Laura Kajetzke / Markus Schroer:
"Space Studies«, in: Stephan Moebius (Hg.): Kultur. Von den Cultural Studies bis zu den Visual Studies. Eine Einführung, Bielefeld 2012, S. 196-215; Jörg Döring / Tristan Thielmann: „Einleitung: Was lesen wir im Raume? Der Spatial Turn und das geheime Wissen der Geographen«, in: Jörg Döring / Tristan Thielmann (Hg.): Spatial Turn. Das Raumparadigma in den Kultur- und Sozialwissenschaften, Bielefeld 2008, S. 7-45. Mit speziellem Blick auf die Geschichtswissenschaft vgl. Rau: Räume, S. 107-121.

99 Gabriela B. Christmann: "Das theoretische Konzept der kommunikativen Raum(re)konstruktion«, in: Gabriela B. Christmann (Hg.): Zur kommunikativen Konstruktion von Räumen. Theoretische Konzepte und empirische Analysen, Wiesbaden 2016, S. 89-117; Geppert / Jensen / Weinhold: „Verräumlichung«.

100 Rau: Räume, S. 53-55. Die naturphilosophischen, mathematischen und physikalischen Raumtheorien von Euklid bis Einstein werden auch in sozial- und kulturwissenschaftlichen Schriften zum Raum immer wieder gerne historisch abgeschritten. Dies beruht auf der (fragwürdigen) Annahme, dass sie die Raumvorstellungen der jeweiligen Zeit geprägt oder doch maßgeblich beeinflusst hätten, oder möglicherweise auch nur darauf, dass die Theoriegeschichte besser aufgearbeitet und zugänglicher ist als die schlecht erforschte breitere Geschichte der Raumvorstellungen; vgl. Wolfgang Göderle: Zensus und Ethnizität. Zur Herstellung von Wissen über soziale Wirklichkeiten im Habsburgerreich zwischen 1848 und 1910, Göttingen 2016, S. 108. Plausibler erscheint, dass sich verändernde Praktiken und Techniken der Raumnutzung und die mit ihnen einhergehenden Verschiebungen in der Wahrnehmung zur Entwicklung neuer Raumtheorien anregten; vgl. Geppert / Jensen / Weinhold: »Verräumlichung«, S. 43-47.

101 Löw: Raumsoziologie, S. 66.

102 Andreas Pott / Alexandra Budke / Detlef Kanwischer: "Internet, Raum und Gesellschaft. Zur Untersuchung eines dynamischen Verhältnisses«, in: Alexandra Budke / Detlef Kanwischer / Andreas Pott (Hg.): Internetgeographien. Beobachtungen zum Verhältnis von Internet, Raum und Gesellschaft, Stuttgart 2004, S. 9-20, hier S. 13.

103 Vgl. Bruno Latour: Das Parlament der Dinge. Für eine politische Ökologie, Frankfurt am Main 2001, S. 302: „Weil die Natur keinen besonderen Bereich der Realität darstellt, sondern Resultat einer politischen Aufteilung ist, einer Verfassung, durch die das Objektive und Unbestreitbare vom Subjektiven und Strittigen geschieden wird«.

104 Cancik: »Verwaltung«, S. 29.

105 Vgl. Jon Mathieu: Die Alpen. Raum - Kultur - Geschichte, Stuttgart 2015.

106 Cancik: »Verwaltung«, S. 35; vgl. Miggelbrink: »(Un-)Ordnung«, S. 80. 107 Löw: Raumsoziologie, S. 66; vgl. Werlen: »Körper«, S. 379-380.

108 Löw: Raumsoziologie, S. 154

109 Dies in Anknüpfung an Reinhard Kreckel: Politische Soziologie der sozialen Ungleichheit, Frankfurt am Main 1992, S. 77.

110 Schroer: Räume, S. 90-106; Löw: Raumsoziologie, S. 210-218.

111 Robert David Sack: Human Territoriality. Its Theory and History, Cambridge 1986, S. 32-33.

112 Henri Lefebvre: La production de l'espace, Paris 1974; vgl. Rau: Räume, S. 47-49.

113 Löw: Raumsoziologie, S. 131.

114 Löw: Raumsoziologie, S. 161-166.

115 Rau: Räume, S. 164-171.

116 Schroer: Räume, S. 276-296.

117 Löw: Raumsoziologie, S. 198-203; vgl. Rau: Räume, S. 64-65.

118 Benz: Staat, S. 83-88; Pierson: Modern State, S. 12-14; vgl. Werlen: »Körper«, S. 381. 
119 Paul Alliès: L'invention du territoire, Grenoble 1980; vgl. Almut Höfert: "States, Cities, and Citizens in the Later Middle Ages«, in: Quentin Skinner / Bo Stråth (Hg.): States and Citizens. History, Theory, Prospects, Cambridge 2003, S. 63-75, hier S. 64.

120 Paul Claval: Les espaces de la politique, Paris 2010, S. 160-161; Arnault Skornicki: "Constructions pratiques et symboliques des frontières politiques«, in: Antonin Cohen / Bernard Lacroix / Philippe Riutort (Hg.): Nouveau manuel de science politique, Paris 2009, S. 74-85, hier S. 75-76; Wolfgang Reinhard: „Einführung: Moderne Staatsbildung - eine ansteckende Krankheit?«, in: Wolfgang Reinhard / Elisabeth Müller-Luckner (Hg.): Verstaatlichung der Welt? Europäische Staatsmodelle und außereuropäische Machtprozesse, München 1999, S. VII-XIV, hier S. IX.

121 Exemplarisch vgl. David Gugerli / Daniel Speich: Topografien der Nation. Politik, kartografische Ordnung und Landschaft im 19. Jahrhundert, Zürich 2002.

122 Dirk Kretzschmar: »Region oder Imperium? Zur Semantik von Geopolitik, Raum und Kultur in Russland «, in: Rudolf Maresch / Niels Werber (Hg.): Raum - Wissen - Macht, Frankfurt am Main 2002, S. 263-286, hier S. 264-265.

123 Eine nähere Auseinandersetzung mit den umfangreichen Debatten um das Verhältnis zwischen sStaatı und `Nation`, insbesondere auch zu jenen um die Unterscheidung zwischen `Nationalstaatı und >Imperium`, unterbleibt hier mit Bedacht. Die für die Fragestellungen dieses Bandes zentralen Aspekte von Staatlichkeit liegen abseits der Hauptfragen dieser Diskussionen.

124 Cancik: „Verwaltung», S. 46; Geppert / Jensen / Weinhold: „Verräumlichung", S. 35-36, 40-41.

125 Lorenz Engell: „Wege, Kanäle, Übertragungen. Zur Einführung», in: Claus Pias et al. (Hg.): Kursbuch Medienkultur. Die maßgeblichen Theorien von Brecht bis Baudrillard, Stuttgart 1999, S. 126-133, hier S. 128.

126 Harold A. Innis: „Das Problem des Raumes« [1951], in: Harold A. Innis: Kreuzwege der Kommunikation. Ausgewählte Texte, hg. von Karlheinz Barck, Wien 1997, S. 147-181, hier S. 179.

127 Wolfgang Hagen: "Globale Gegenwartsversessenheit. Annäherungen an Harold A. Innis und seine Theorie einer Medien-Raumzeit", in: Rudolf Maresch / Niels Werber (Hg.): Raum - Wissen - Macht, Frankfurt am Main 2002, S. 193-213, hier S. 204.

128 Christoph Kleinschmidt: „Einleitung: Formen und Funktionen von Grenzen«, in: Christoph Kleinschmidt / Christine Hewel (Hg.): Topographien der Grenze. Verortungen einer kulturellen, politischen und ästhetischen Kategorie, Würzburg 2011, S. 9-21, hier S. 9.

129 Peter Weichhardt: „Territorialität, Identität und Grenzerfahrung«, in: Peter Haslinger (Hg.): Grenze im Kopf. Beiträge zur Geschichte der Grenze in Ostmitteleuropa, Frankfurt am Main 1999, S. 19-30.

130 Katrin Lehnert / Lutz Vogel: „Einleitung. Kleinräumige Mobilität und Grenzwahrnehmung im 19. Jahrhundert«, in: Katrin Lehnert / Lutz Vogel (Hg.): Transregionale Perspektiven. Kleinräumige Mobilität und Grenzwahrnehmung im 19. Jahrhundert, Dresden 2011, S. 9-22, hier S. 15.

131 Andreas Rutz: „Grenzüberschreitungen im deutsch-niederländischfranzösischen Grenzraum«, in: Christine Roll / Frank Pohle / Matthias Myrczek (Hg.): Grenzen und Grenzüberschreitungen. Bilanz und Perspektiven der Frühneuzeitforschung, Köln 2010, S. 217-222, hier S. 221.

132 Exemplarisch vgl. Sebastian Scharte: „Vom Bestreben, auch an den äußersten Westmarken nach Kräften der vaterländischen Sache zu dienen. Die rheinpreußischen Grenzkreise Eupen und Malmedy 1815-1914«, in: Katrin Lehnert / Lutz Vogel (Hg.): Transregionale Perspektiven. Kleinräumige Mobilität und Grenzwahrnehmung im 19. Jahrhundert, Dresden 2011, S. 191-203.
133 Lars Behrisch: „Vermessen, Zählen, Berechnen des Raums im 18. Jahrhundert«, in: Lars Behrisch (Hg.): Vermessen, Zählen, Berechnen. Die politische Ordnung des Raums im 18. Jahrhundert, Frankfurt am Main 2006, S. 7-25. Für die Hinwendung zur Erforschung des Raumwissens wird gelegentlich die Bezeichnung stopographical turn in Unterscheidung vom sspatial turn gebraucht; vgl. Stephan Günzel: »Raum - Topographie - Topologie«, in: Stephan Günzel (Hg.): Topologie. Zur Raumbeschreibung in den Kultur- und Medienwissenschaften, Bielefeld 2007, S. 13-29, hier S. 18-21.

134 Anton Tantner: Ordnung der Häuser, Beschreibung der Seelen. Hausnummerierung und Seelenkonskription in der Habsburgermonarchie, Innsbruck 2007. Zur einheitlichen Ordnung der Verwaltungsräume als Voraussetzung für spätere Volkszählungen in der Habsburgermonarchie vgl. Göderle: Zensus und Ethnizität, S. 77-86.

135 Allerdings ist darauf hinzuweisen, dass die Erhebung und Erfassung von Wissen nicht selten auch dann gepflogen wurde, wenn eine praktische Verwertbarkeit eher für die Zukunft vermutet wurde, als aktuell gegeben zu sein; vgl. Elke Hartmann: Die Reichweite des Staates. Wehrpflicht und moderne Staatlichkeit im Osmanischen Reich 1869-1910, Paderborn 2016, S. 348.

136 Scott: Seeing Like a State.

137 Kerstin Brückweh: Menschen zählen. Wissensproduktion durch britische Volkszählungen und Umfragen vom 19. Jahrhundert bis ins digitale Zeitalter, Berlin 2015; Michael C. Schneider: Wissensproduktion im Staat. Das königlich preußische statistische Bureau 1860-1914, Frankfurt am Main 2013; Silvana Patriarca: Numbers and Nationhood. Writing Statistics in Nineteenth-Century Italy, Cambridge 1996; Alain Desrosières: La politique des grands nombres. Histoire de la raison statistique, Paris 1993.

138 Walter Brunner: „Historische Bodendokumentation und die Anfänge der Katastralvermessung in Mitteleuropa. Ein Überblick«, in: Mitteilungen des Steiermärkischen Landesarchivs 47 (1997), S. 89-105; Roger J. P. Kain / Elizabeth Baigent: The Cadastral Map in the Service of the State. A History of Property Mapping, Chicago 1992.

139 Stephan Günzel: "Spatial Turn - Topographical Turn - Topological Turn. Über die Unterschiede zwischen Raumparadigmen«, in: Jörg Döring / Tristan Thielmann (Hg.): Spatial Turn. Das Raumparadigma in den Kultur- und Sozialwissenschaften, Bielefeld 2008, S. 219-237, hier S. 230-234; Jean-Pierre Le Bourhis: »Quadriller le territoire. La cartographie au service de l'action publique contre les risques naturels«, in: Olivier Ihl / Martine Kaluszynski / Gilles Pollet (Hg.): Les sciences de gouvernement, Paris 2003, S. 157-167; Gugerli / Speich: Topografien; Matthew H. Edney: Mapping an Empire. The Geographical Construction of British India, 1765-1843, Chicago 1997; Gilles Palsky: Des chiffres et des cartes. Naissance et développement de la cartographie quantitative française au XIXe siècle, Paris 1996.

140 Raphael: »Staat im Dorf«, S. 47-48.

141 Rainer Polley: »Kollegialprinzip und Geschäftsgang im 19. Jahrhundert. Eine verfassungs- und verwaltungsgeschichtliche Fallstudie zur Aktenkunde«, in: Archiv für Diplomatik, Schriftgeschichte, Siegel- und Wappenkunde 42 (1996), S. 445-488, hier S. 446. 


\section{Abstract}

A particular relationship with space, usually called territoriality, is one of the essential characteristics of the modern state. This statement was long considered a commonplace. Recent debates, however, have raised new fundamental questions about both space and the state which require a re-examination of both terms, and thus of the connections between them as well. This introduction maps out some of the terminological and theoretical ground for research into these questions. We successively examine the conceptual history of the state, of public administration, and of space, pointing out reifying uses of all three notions which have been repudiated in theoretical debates but remain influential in many historiographical accounts, as well as in popular discourse. We highlight alternative approaches suggested by newer authors. In particular, we describe both the state and administration in terms of assemblages of people, institutions, and objects. Given that this perspective is also used in some current socio-cultural theories of space, we conclude that states and administrations not only exist in space, use space, and create and shape spaces, but that they are themselves spaces and can be analyzed using the methodological tools which apply to spaces of any kind.

\section{About the Authors}

Stefan Nellen leads the Historical Analysis Services of the Swiss Federal Archives, which has a focus on history of administration. He holds a Ph.D. in History from the University of Basel. His research interests include history of administration, cultural techniques, governmentality and history of madness and psychiatry. He has published widely on the history of public administration; see for example "Die Akte der Verwaltung. Zu den administrativen Grundlagen des Rechts«, in: Marcus Twellmann (ed.): Wissen, wie Recht ist. Bruno Latours empirische Philosophie einer Existenzweise, Konstanz 2016, p. 65-91; „Das Wesen der Registratur. Zur Instituierung des Dokumentarischen in der Verwaltung«, in: Renate Wöhrer (ed.): Wie Bilder Dokumente wurden. Zur Genealogie einer Darstellungspraxis, Berlin 2015 , p. $225-248$

THOMAS Stockinger is a post-doctoral university assistant for Austrian History in the Institute of Austrian Historical Research at the University of Vienna. His research focuses on the political and administrative history of the Habsburg Monarchy in the 19th century, with special attention to the interactions between population and public institutions in rural areas, including elections and local administration. He also works on the history of historical research and historiography. 